\title{
DISPERSED ORGANIC MATTER ANALYSIS AND DISTRIBUTION IN THE SEQUENCE STRATIGRAPHIC FRAMEWORK OF THE SOUTHEASTERN, PARANÁ BASIN, BRAZIL
}

\section{MARCUS VINICIUS BERAO ADE ${ }^{1 *}$ AND LUIZ ANTONIO TRINDADE ${ }^{2}$}

1 Universidade do Estado do Rio de Janeiro, Faculdade de Geologia, Departamento de Estratigrafia e Paleontologia, Rua São Francisco Xavier, 524, 20550-900, Rio de Janeiro, RJ, Brazil.marcusade@uerj.br

2 Petrobras/EXP/AEXP-T/OP, Edificio Ventura, Torre Leste, 17 andar, Av. República do Chile, 330 - Centro, Rio de Janeiro - RJ, 20031-170, Brazil. luiz.trindade@petrobras.com.br

*Corresponding Author, marcusade@uerj.br

Received on 18 September 2017

Received in revised form on 27 October 2017

Accepted on 28 October 2017

Editor: Maria Virginia Alves Martins, Universidade do Estado do Rio de Janeiro, Brazil

\section{Abstract}

The study area is located on southeastern boundary of the intracratonic Paraná Basin, in the northeastern region of Rio Grande do Sul State, Brazil. The study of organic matter characterization and its distribution in relation to the sequence stratigraphy method is of great importance for the identification of possible target or inappropriate areas for exploration on the eastern border of this basin.

Three boreholes were analysed in this work and sample sets were collected from the sedimentary section covering a Permian stratigraphic interval, from sequences II, III and IV (between the base of Rio Bonito Formation and the top of Teresina Formation). Results of this work evidence that: the concentration and composition of the dispersed organic matter (DOM) in the sedimentary package is associated with production, dilution, preservation and degradation of the organic matter; the organic fragments preserved in sedimentary rocks are predominantly composed of a mixture of particles of the inertinite group and lesser amounts from the vitrinite group, both derived from terrestrial plants; consequently, the hydrogen and oxygen indexes are low; since the kerogen type is II and IV, it does not contribute to the generation of oil. The organic matter distribution within the analysed sequence stratigraphic shows the following general trend or behaviour: 1) total organic carbon, hydrogen index and vitrinite group contents are relatively high in the rocks of the transgressive system but are lower in rocks of the lowstand and highstand systems; 2) the Inertinite group contents show the opposite trend, with the highest concentrations in the rocks of the lowstand and the highstand systems.

The results of this work also evidence that in the upper strata of Rio Bonito Formation the OM is immature but should have reached the oil window (catagenesis) in the strata located below this formation.

Keywords: Sequence Stratigraphy. Organic Petrography. Pyrolysis Rock-Eval. Hydrocarbon generation. Coal. 


\section{Introduction}

Organic matter $(\mathrm{OM})$ studies can be associated with lithostratigraphic and organic facies characterizations. Organic facies are mappable stratigraphic units that are distinguished by their organic matter composition (Jones, 1987). Several organic facies studies have demonstrated that organic deposits found on continental shelves change during regional events from transgressive to regressive phases (Pasley et al., 1991, 1993).

Numerous papers have been written about how organic matter is preserved in the geological record. According to Tyson (1987) and Tyson and Pearson (1991) relatively dysoxic conditions and low or absent sea bottom circulation is necessary to accumulate rich-organic sediments with total organic carbon (TOC) exceeding $1 \%$ (TOC $>1 \%$ ). Tyson and Pearson (1991) noticed that biologic productivity is a secondary factor in the accumulation rate of OM. However, Calvert and Pedersen (1993) defend that biologic productivity and accumulation rate of $\mathrm{OM}$ are the major factors for the occurrence of rich-organic deposits, with dysoxic conditions as secondary factors. According to Parrish (1995), the problem is that both schools (of Tyson and Pearson and Calvert and Pedersen) have based their assumptions on modern oceanographic studies (uniformitarianism vision). Unfortunately, the oxygen content of the ancestral seas is not preserved in the geologic record.

\subsection{The main goals}

This work aims to determine the quality, quantity, organic facies and the maturity degree of the organic material based on petrographic and pyrolysis analyses in three boreholes (5CA-81-RS 7-GT-10-RS and 2-TG-96-RS) collected in Paraná Basin, Brazil. It also aims to determine the capacity for hydrocarbon generation.

\section{Study area}

The Paraná Basin is a large intracratonic Paleozoic basin $\left(1,700,000 \mathrm{~km}^{2}\right)$, located in central-southeastern South America continent with portions of the basin found in Brazil, Uruguay, Argentina and Paraguay (Fig. 1) (Zalán et al., 1990). Paraná Basin contains upwards to 8,000-meter thickness of sedimentary and igneous rocks.

Stratigraphic studies of the Paraná Basin (PB) began, in Brazil, with the work of White (1908). Since then, several lithostratigraphic works were published, but only after 1974 comprehensive compilations of the stratigraphic column of PB were published by Schneider et al. (1974) and Zalán et al.
(1990). Later Milani et al. (2007) (Fig. 2) modified this stratigraphic framework.

According to Zalán et al. (1990) and Milani et al. (2007), the Paraná Basin was established over a cooling continental crust after the Brasiliano Orogenic Cycle (700-450 million years). The resulting thermal subsidence, which began between the Cambrian and Ordovician Periods, caused sedimentation. This started at the Silurian and ended at the Cretaceous with several breaks or unconformities in the sedimentary record.

The Itararé and Guatá Groups within the Paraná Basin, in the northeastern area of the Rio Grande do Sul State, studied by several authors (Holz et al., 1986; Piccoli et al., 1986; Lavina and Lopes, 1987), contain a major lithostratigraphic boundary representing a transgressiveregressive cycle.

Milani et al. (2007), applying Sequence Stratigraphic concepts to Paraná Basin, identified six second order sequences and named them from the bottom to the top as: Ordovician-Silurian, Devonian, early Carboniferous, Triassic, late Triassic, early Jurassic, Cretaceous and late Cretaceous. The sedimentary section in this study is situated in early Carboniferous to Triassic Sequence inside the lithostratigraphic Itararé, Guatá and Passa Dois Groups. The stratigraphic sequence column originally done by $\mathrm{Holz}$ (1997) and modified by Etgeton (1997) was adopted in this work. Both researches have used the traditional sequence stratigraphic nomenclature, and proposed a subdivision of the Milani's second order sequence (Carboniferous to early Triassic Sequence) (Fig. 3). The rocks in this study covered the Permian stratigraphic interval from sequences II, III and IV (from the base of Rio Bonito Formation to the top of Teresina Formation).

\section{Material and methods}

This study is based on results obtained from samples from three boreholes: 5-CA-81-RS (latitude 29॰52'19.8” S, longitude 50॰53’00” W), 7-GT-10-RS (latitude 29॰51'49.8” S, longitude 50 $566^{\prime} 52.5^{\prime \prime} \mathrm{W}$ ) and 2-TG-96-RS (latitude 29०44'1.57” S, longitude 50॰04'15” W, collected in Paraná Basin, Brazil (Fig. 1). The samples that were selected and analysed were positioned between the sequences II and IV (Rio Bonito and Teresina Formations).

After a lithologic macroscopic description of each sample, the samples were split in two subsamples. One subsample was analysed for TOC content and pyrolysis using a WR-12 LECO apparatus and a Rock-Eval III apparatus, respectively. 


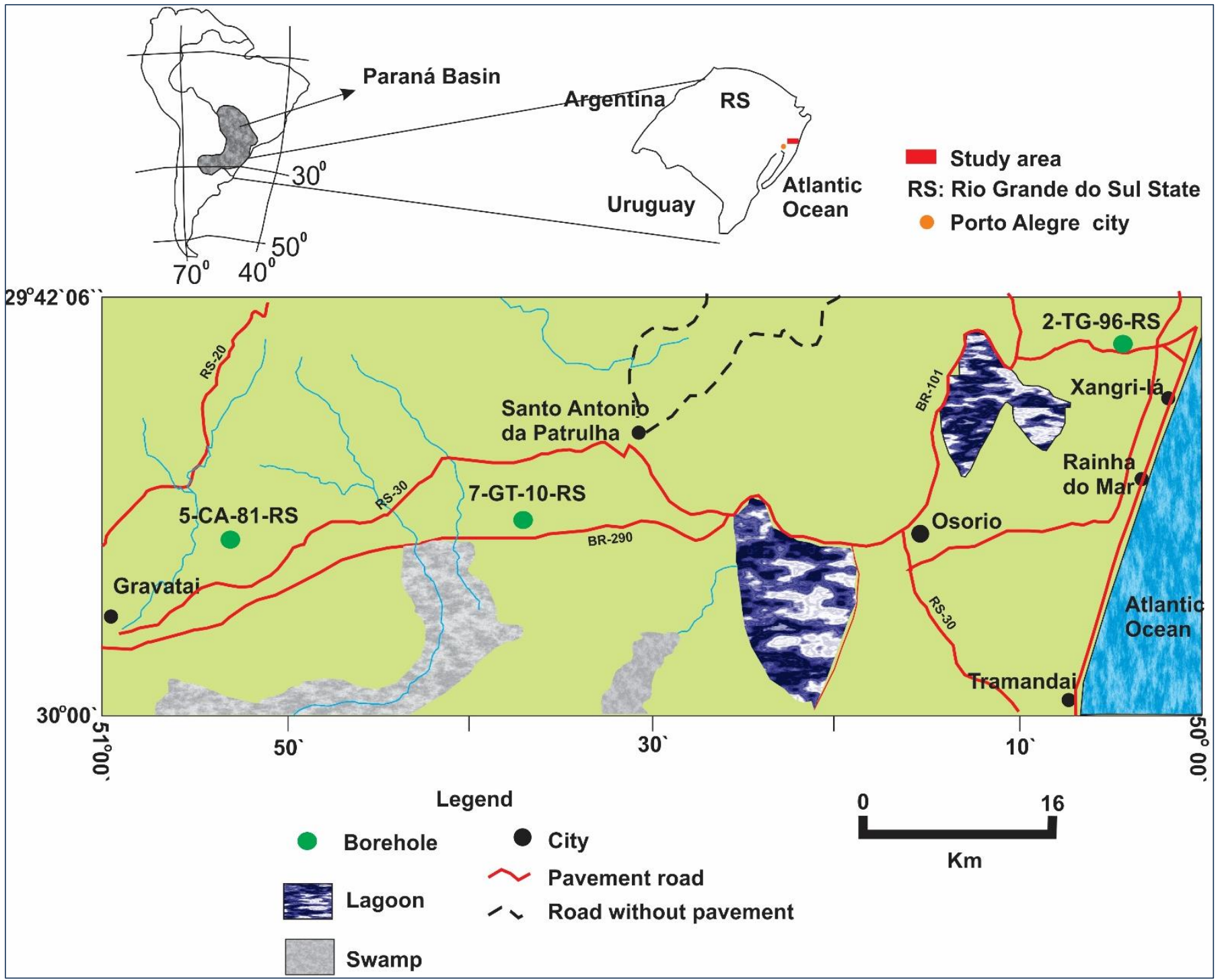

Fig. 1. Geographic map and Wells 5-CA-8-RS, 7-GT-10-RS and 2-TG-96-RS location (according to the location map of Borda Leste Project, DNPM/CPRM, 1986).

Pyrolysis Rock-Eval III is a method, where solid, liquid and gas materials are degraded thermally or pyrolysed without oxygen (Espitalié et al., 1985). Kerogen has been characterized as type I (very oil prone), type II (oil prone), type III (gas prone) (van Krevelen, 1961, in Tissot and Welte, 1978) and type IV (inert) (Brooks, 1981).

The second fraction was used to make two-polished sections for whole rocks and concentrated organic matter measurements. This study was conducted using incident white light (halogen, 12V, $100 \mathrm{~W}$ ) and ultraviolet light (HBO $100 \mathrm{~W}$ ) on a Leitz Orthoplan-Pol microscope equipped with 50x 0.85 OEL P objectives and Periplan 10x oculars.

\subsection{Dispersed organic matter (DOM)}

Microscopic studies of dispersed organic matter (DOM) in source rocks have been applied mainly in oil and gas exploration aiming to characterize and to identify several organic constituents, and to determine the degree of maturity of OM (Teichmüller, 1986).

The nomenclature of DOM was based on the international standardized coal material classification (Stach et al., 1975; Taylor et al., 1998). This study also analyses the unstructured organic matter (bitumen and organic-mineral association). 
RESEARCH PAPER

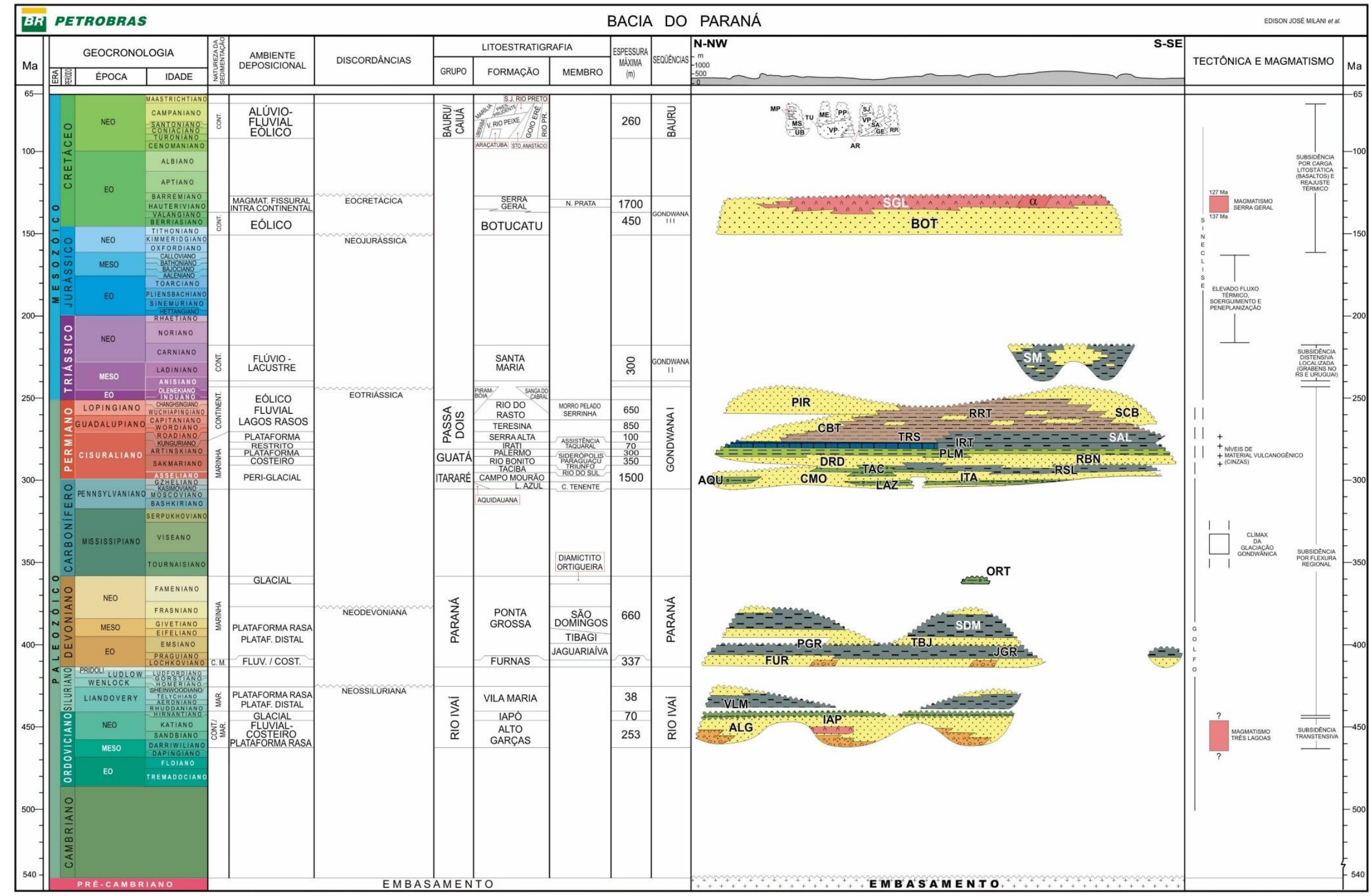

Fig. 2. Lithostratigraphy and sequence stratigraphy column of Paraná Basin (Milani et al., 2007). 


\begin{tabular}{|c|c|c|c|}
\hline \multirow{2}{*}{ Lithostratigraphy } & \multirow{2}{*}{$\begin{array}{l}\text { Sequence } \\
2^{\text {nd }} \text { order }\end{array}$} & \multicolumn{2}{|c|}{ Sequence $3^{\text {rd }}$ Order } \\
\hline & & Holz (1997) & Etgeton (1997) \\
\hline Rio do Rastro Fm & \multirow{7}{*}{ 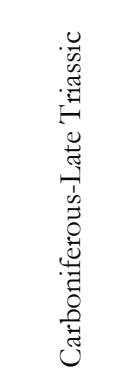 } & \multirow{3}{*}{ ? ? ? ? } & SD 4 \\
\hline Teresina Fm & & & \multirow{2}{*}{ SD 3} \\
\hline Serra Alta Fm & & & \\
\hline Irati Fm & & Sequence IV & \multirow{3}{*}{ SD 2} \\
\hline Palermo Fm & & Sequence III & \\
\hline Rio Bonito Fm & & Sequence II & \\
\hline Itarare Gp & & Sequence I & SD 1 \\
\hline
\end{tabular}

Fig. 3. Lithostratigraphy and sequence stratigraphy relationship according Milani et al. (2007), Holz (1997) and Etgeton (1997). Legend: SD- Sequence Depositional. Fm - Formation; Gp Group.

DOM studies have not been international standardized. Organic concentration methods and organic particle terminology vary between different laboratories. This problem has been discussed in the international meetings (International Committee for Coal and Organic PetrologyICCP and The Society for Organic Petrology-TSOP) without resolution, until the recent proposals by Teerman et al. (1995) and Hutton et al. (1999). For DOM analysis, the samples were concentrated by dissolving minerals in successive treatments of $\mathrm{HCI}, \mathrm{HF}$ and $\mathrm{ZnCl}$ with a 1.9gms/cc density. DOM materials were classified according to suggestions of the International Committee for Coal and Organic Petrology (ICCP, 1993; Hutton et al., 1999) considering:

1) Structured Organic Matter: a) Vitrinite; b) Inertinite; c) Liptinite, Sporinite, Cutinite, Resinite, Telalginite, Lamalginite and Liptodetrinite; d) Migrabitumen and e) Zooclastics.

2) Unstructured Organic Matter: a) Bituminite; b) Mineral Organic Matter.

Quantitative and qualitative evaluations in white and fluorescence light were conducted on both the whole rock and organic concentrates. For quantitative petrographic evaluation the following percentage categories were used: Very Abundant $(\mathrm{VAb})>90 \%$; Abundant $(\mathrm{Ab}) 90-70 \%$; Average (Av) $69-40 \%$; Low (L) $39-10 \%$; Very Low (VL) $<10 \%$; Trace $(\operatorname{Tr})<6$ particles and; Not Observed (NO).

\section{Results}

\subsection{Dispersed Organic Matter (DOM) Characterization}

Microscopic results of the organic constituents of all sedimentary sequences are shown in Tables 1,2 and 3 . In the three analysed boreholes, independent of their stratigraphic position, the inertinite and the vitrinite groups are generally the predominant material, with some exceptions in the Rio Bonito and in the Iratí Formations.

Sample TG-9825 from the Rio Bonito Formation shows a predominance of the liptinite group. Samples TG-9826, TG-9827 and TG-9828, of the borehole 2-TG-96-RS (Table 1) have organic concentrations between the inertinite and the liptinite groups.

Vitrinite predominance occurs in samples TG-9817 and TG-9818 (Iratí Formation; Table 1) and similar concentrations of the vitrinite and the inertinite groups were observed in the samples GT-9 and GT-10 (Table 2). In the southwestern portion of the study area in the Iratí Formation (borehole 5-CA-81-RS), the composition of organic matter changes to a predominance of the liptinite group (samples CA-9 and CA-11; Table3).

\subsection{Results from pyrolysis analysis}

Pyrolyzed samples had TOC values $>0.5 \%$. The pyrolysis results are shown in Tables 4, 5 and 6 .

In general, the samples in the study area present low hydrogen indexes $(<300 \mathrm{mg} \mathrm{HC} / \mathrm{g}$ TOC) and low oxygen indexes ( $<130 \mathrm{mg} \mathrm{CO} / \mathrm{g}$ TOC). These results shown in Fig. 4 coupled with the microscopic study, allow the characterization of the kerogen for the majority of the samples as type III, with some samples of coaly-shale and coal, as type IV.

Some of the samples in Sequence II (9613693.92, 9613696.33, 9613697.17, TG-9828 and TG9839, Table 4 and; 9613635.52, Table 6 ) belonging to the transgressive system tract and lowstand system tract (Rio Bonito Formation), with HI values between 140 and 270 (mg HC/g TOC) (Fig. 4), could be classified, using the pyrolysis analysis, as kerogen Types II or I.

However, the results of the microscopic study of the organic content of these samples indicate that they have similar concentrations of the inertinite (Av) and the liptinite (Av) groups.

Further characterization, allows to verify that the kerogen derives of a mixture of OM type II and OM type IV. The pyrolysis analysis on the above mixture of kerogens result in a type III kerogen. The low values of the OI and HI are related to large concentrations of inertinite.

Sequence III as shown in the diagram of Fig. 4 has only samples in the top of the highstand system tract 3 (HST 3). The other system tract in the Sequence III has a dominant arenaceous characteristic (boreholes 5-CA-81-RS and 2-TG96-RS) and have low values of TOC (<0.5\%). 
Tab. 1. Petrographic composition analysis for sample of disperse organic matter on whole rock and organic concentrate in reflected white light and fluorescence light. Legend: Depth (m); V- Vitrinite; I- Inetinite; L- Liptinite; Esp- Sporinite; Cut- Cutinite; Res- Resinite; Tel- Telalginite; Lam- Lamalginite; Mig- Migrabitumen; Lip- Liptodetrinite; MOM- Mineral Organic Matter; Bet- Bituminite; VA- Very abundant (>90\%); Ab- Abundant (90-70\%); Av- Average (69-40\%); L -Low (39-10\%); VL- Very low (<10\%); Tr- Trace $(<6$ ponts); NO- Not Observed.

\begin{tabular}{|c|c|c|c|c|c|c|c|c|c|c|c|c|c|}
\hline \multirow{3}{*}{\multicolumn{2}{|c|}{$\begin{array}{l}\text { Samples } \\
\text { reference }\end{array}$}} & \multicolumn{12}{|c|}{ Borehole 2-TG-96-RS } \\
\hline & & \multicolumn{10}{|c|}{ Structured Organic Matter } & \multirow{2}{*}{\multicolumn{2}{|c|}{$\begin{array}{l}\text { Unstructured } \\
\text { Organic Matter }\end{array}$}} \\
\hline & & \multicolumn{3}{|c|}{ Macerals Group } & \multicolumn{6}{|c|}{ Macerals Group of Liptinite } & \multirow[t]{2}{*}{ Mig } & & \\
\hline Sample & Depth & V & I & $\mathbf{L}$ & Esp & Cut & Res & Tel & Lam & Lip & & MOM & Bet \\
\hline \multicolumn{14}{|c|}{ Sequence IV } \\
\hline TG9848 & 691.1 & $\mathrm{~L}$ & $\mathrm{Av}$ & VL & $\operatorname{Tr}$ & $\operatorname{Tr}$ & $\mathrm{NO}$ & $\operatorname{Tr}$ & $\mathrm{Vl}$ & $\mathrm{Ab}$ & $\mathrm{L}$ & $\mathrm{NO}$ & $\mathrm{VL}$ \\
\hline TG9807 & 706.4 & $\operatorname{Tr}$ & VA & $\mathrm{NO}$ & -- & -- & -- & -- & -- & -- & -- & $\mathrm{NO}$ & $\operatorname{Tr}$ \\
\hline TG9809 & 716.4 & $\mathrm{NO}$ & VA & VL & $\mathrm{NO}$ & $\mathrm{NO}$ & $\mathrm{NO}$ & $\mathrm{NO}$ & $\mathrm{NO}$ & VA & $\mathrm{NO}$ & $\mathrm{NO}$ & $\mathrm{NO}$ \\
\hline TG9810 & 719.4 & $\operatorname{Tr}$ & VA & $\operatorname{Tr}$ & $\mathrm{NO}$ & $\mathrm{NO}$ & $\mathrm{NO}$ & NO & $\operatorname{Tr}$ & $\operatorname{Tr}$ & $\mathrm{NO}$ & NO & $\mathrm{NO}$ \\
\hline TG9811 & 720.1 & $\operatorname{Tr}$ & $\mathrm{Ab}$ & VL & $\operatorname{Tr}$ & $\mathrm{NO}$ & $\mathrm{NO}$ & $\operatorname{Tr}$ & $\mathrm{NO}$ & VA & $\operatorname{Tr}$ & NO & $\mathrm{VL}$ \\
\hline TG9812 & 722.0 & $\mathrm{~L}$ & $\mathrm{Ab}$ & $\operatorname{Tr}$ & $\mathrm{NO}$ & $\mathrm{NO}$ & $\mathrm{NO}$ & No & $\mathrm{NO}$ & $\operatorname{Tr}$ & $\mathrm{NO}$ & $\mathrm{NO}$ & $\mathrm{NO}$ \\
\hline TG9813 & 723.0 & $\mathrm{VP}$ & $\mathrm{R}$ & $\operatorname{Tr}$ & $\mathrm{NO}$ & $\mathrm{NO}$ & $\mathrm{NO}$ & $\mathrm{NO}$ & $\operatorname{Tr}$ & $\operatorname{Tr}$ & NO & $\mathrm{NO}$ & $\mathrm{NO}$ \\
\hline TG9814 & 729.0 & $\mathrm{NO}$ & VA & $\mathrm{NO}$ & -- & -- & -- & -- & -- & -- & -- & $\mathrm{NO}$ & $\mathrm{NO}$ \\
\hline TG9815 & 744.1 & $\mathrm{VL}$ & VA & $\mathrm{VL}$ & NO & $\mathrm{NO}$ & $\mathrm{NO}$ & NO & $\mathrm{NO}$ & VA & NO & $\mathrm{NO}$ & $\operatorname{Tr}$ \\
\hline TG9816 & 794.1 & $\mathrm{~L}$ & $\mathrm{Ab}$ & $\mathrm{NO}$ & -- & -- & -- & -- & -- & -- & -- & $\mathrm{NO}$ & $\mathrm{NO}$ \\
\hline TG9817 & 797.7 & $A v$ & $\mathrm{~L}$ & $\mathrm{NO}$ & -- & -- & -- & -- & -- & -- & -- & NO & $\mathrm{NO}$ \\
\hline TG9818 & 800.9 & $A v$ & $\mathrm{~L}$ & $\operatorname{Tr}$ & $\mathrm{NO}$ & $\mathrm{NO}$ & $\mathrm{NO}$ & $\mathrm{NO}$ & $\operatorname{Tr}$ & $\operatorname{Tr}$ & $\mathrm{NO}$ & $\mathrm{NO}$ & $\operatorname{Tr}$ \\
\hline TG9819 & 803.6 & VL & Av & $\mathrm{L}$ & $\mathrm{L}$ & $\mathrm{NO}$ & $\mathrm{NO}$ & $\mathrm{Tr}$ & $\mathrm{NO}$ & $\mathrm{L}$ & $\mathrm{Ab}$ & $\mathrm{VL}$ & $\mathrm{L}$ \\
\hline TG9820 & 804.9 & VL & $\mathrm{Ab}$ & $\mathrm{L}$ & $\mathrm{NO}$ & $\mathrm{NO}$ & $\mathrm{NO}$ & VL & $\mathrm{NO}$ & VA & $\mathrm{NO}$ & $\mathrm{VL}$ & $\mathrm{NO}$ \\
\hline \multicolumn{14}{|c|}{ Sequence III } \\
\hline TG9821 & 807.3 & $\mathrm{~L}$ & $\mathrm{Ab}$ & $\mathrm{L}$ & $\mathrm{NO}$ & $\mathrm{NO}$ & NO & $\operatorname{Tr}$ & $\mathrm{NO}$ & $\mathrm{NO}$ & VA & $\mathrm{NO}$ & $\mathrm{NO}$ \\
\hline TG9823 & 816.5 & $\mathrm{~L}$ & $\mathrm{Ab}$ & $\mathrm{VL}$ & NO & $\mathrm{NO}$ & $\mathrm{NO}$ & $\operatorname{Tr}$ & $\mathrm{NO}$ & $\operatorname{Tr}$ & $\mathrm{Va}$ & $\mathrm{NO}$ & $\mathrm{NO}$ \\
\hline \multicolumn{14}{|c|}{ Sequence II } \\
\hline TG9824 & 889.1 & $\mathrm{NO}$ & VA & $\operatorname{Tr}$ & $\mathrm{NO}$ & $\operatorname{Tr}$ & $\mathrm{NO}$ & $\mathrm{NO}$ & $\mathrm{NO}$ & $\operatorname{Tr}$ & $\mathrm{NO}$ & $\mathrm{NO}$ & $\mathrm{NO}$ \\
\hline TG9825 & 890.1 & $\mathrm{VL}$ & $\mathrm{L}$ & Av & $\mathrm{VL}$ & $\mathrm{NO}$ & $\mathrm{NO}$ & $\mathrm{NO}$ & Av & $\mathrm{L}$ & $\mathrm{L}$ & $\mathrm{NO}$ & $\mathrm{VL}$ \\
\hline TG9826 & 891.0 & $\mathrm{~L}$ & $\mathrm{Av}$ & Av & $\mathrm{Ab}$ & NO & $\mathrm{NO}$ & $\mathrm{L}$ & $\mathrm{NO}$ & Av & $\mathrm{L}$ & $\mathrm{NO}$ & $\mathrm{VL}$ \\
\hline TG9827 & 891.5 & $\mathrm{~L}$ & $\mathrm{Av}$ & $\mathrm{Av}$ & $\mathrm{Ab}$ & $\mathrm{NO}$ & $\mathrm{NO}$ & $\mathrm{VL}$ & $\mathrm{NO}$ & $\mathrm{L}$ & $\mathrm{VL}$ & $\mathrm{NO}$ & $\mathrm{VL}$ \\
\hline TG9828 & 910.0 & $\mathrm{VL}$ & $\mathrm{Av}$ & $\mathrm{Av}$ & $\mathrm{Ab}$ & $\operatorname{Tr}$ & $\mathrm{NO}$ & $\operatorname{Tr}$ & $\operatorname{Tr}$ & $\mathrm{L}$ & $\mathrm{NO}$ & $\mathrm{NO}$ & $\operatorname{Tr}$ \\
\hline TG9833 & 959.2 & $\mathrm{~L}$ & $\mathrm{Ab}$ & $\mathrm{VL}$ & $\operatorname{Tr}$ & $\mathrm{NO}$ & $\mathrm{NO}$ & $\operatorname{Tr}$ & $\mathrm{NO}$ & VA & $\mathrm{NO}$ & $\mathrm{VL}$ & $\mathrm{VL}$ \\
\hline TG9834 & 965.7 & $\mathrm{~L}$ & Av & $\operatorname{Tr}$ & $\mathrm{NO}$ & $\mathrm{NO}$ & $\mathrm{NO}$ & $\operatorname{Tr}$ & NO & $\mathrm{NO}$ & NO & NO & $\mathrm{NO}$ \\
\hline TG9835 & 967.7 & $\mathrm{~L}$ & Av & $\operatorname{Tr}$ & $\operatorname{Tr}$ & $\mathrm{NO}$ & $\mathrm{NO}$ & $\operatorname{Tr}$ & $\mathrm{NO}$ & $\operatorname{Tr}$ & $\mathrm{NO}$ & $\mathrm{NO}$ & $\mathrm{NO}$ \\
\hline TG9836 & 971.6 & $\operatorname{Tr}$ & $\mathrm{Ab}$ & $\mathrm{L}$ & $\mathrm{Tr}$ & $\mathrm{NO}$ & $\mathrm{NO}$ & $\mathrm{NO}$ & $\mathrm{L}$ & VA & $\mathrm{NO}$ & $\mathrm{VL}$ & $\mathrm{NO}$ \\
\hline TG9837 & 980.3 & $\mathrm{VL}$ & $\mathrm{Ab}$ & $\mathrm{L}$ & $\mathrm{L}$ & $\mathrm{NO}$ & $\mathrm{NO}$ & $\mathrm{Av}$ & $\mathrm{NO}$ & $\mathrm{Av}$ & $\mathrm{NO}$ & $\operatorname{Tr}$ & $\operatorname{Tr}$ \\
\hline TG9838 & 985.1 & $\operatorname{Tr}$ & $\mathrm{Ab}$ & $\mathrm{L}$ & $\operatorname{Tr}$ & $\mathrm{NO}$ & $\mathrm{NO}$ & $\mathrm{Ab}$ & $\mathrm{NO}$ & $\mathrm{L}$ & NO & $\mathrm{Tr}$ & $\mathrm{NO}$ \\
\hline TG9839 & 988.0 & $\mathrm{~L}$ & $\mathrm{Av}$ & $\mathrm{L}$ & $\mathrm{Ab}$ & $\operatorname{Tr}$ & $\mathrm{NO}$ & $\mathrm{L}$ & $\mathrm{NO}$ & $\operatorname{Tr}$ & NO & $\operatorname{Tr}$ & $\mathrm{VL}$ \\
\hline
\end{tabular}


Tab. 2. Petrographic composition analysis for sample of disperse organic matter on the whole rock and organic concentrate in reflected white light and fluorescence light. Legend: Depth (m); V- Vitrinite; I- Inetinite; L- Liptinite; Esp- Sporinite; Cut- Cutinite; Res- Resinite; Tel- Telalginite; Lam- Lamalginite; Mig- Migrabitumen; Lip- Liptodetrinite; MOM- Mineral Organic Matter; Bet- Bituminite; VA- Very abundant (>90\%); Ab- Abundant (90-70\%); Av- Average (69-40\%); L -Low (39-10\%); VL- Very low $(<10 \%)$; Tr- Trace $(<6$ ponts); NO- Not Observed.

\begin{tabular}{|c|c|c|c|c|c|c|c|c|c|c|c|c|c|}
\hline \multirow{3}{*}{\multicolumn{2}{|c|}{$\begin{array}{l}\text { Samples } \\
\text { reference }\end{array}$}} & \multicolumn{12}{|c|}{ Borehole 7-GT-10-RS } \\
\hline & & \multicolumn{10}{|c|}{ Structured Organic Matter } & \multirow{2}{*}{\multicolumn{2}{|c|}{$\begin{array}{c}\text { Unstructured } \\
\text { Organic Matter }\end{array}$}} \\
\hline & & \multicolumn{3}{|c|}{ Macerals Group } & \multicolumn{6}{|c|}{ Macerals Group of Liptinite } & \multirow[t]{2}{*}{ Mig } & & \\
\hline Sample & Depth & $\mathbf{V}$ & I & $\mathbf{L}$ & Esp & Cut & Res & Tel & Lam & Lip & & MOM & Bet \\
\hline \multicolumn{14}{|c|}{ Sequence IV } \\
\hline GT1 & 255.9 & $\mathrm{~L}$ & $\mathrm{Ab}$ & $\mathrm{L}$ & $\mathrm{L}$ & $\operatorname{Tr}$ & $\mathrm{NO}$ & $\mathrm{VP}$ & NO & $\mathrm{Ab}$ & $\operatorname{Tr}$ & $\operatorname{Tr}$ & $\operatorname{Tr}$ \\
\hline GT3 & 289.5 & $\mathrm{~L}$ & $\mathrm{Ab}$ & VP & $\mathrm{Av}$ & $\mathrm{NO}$ & NO & $\mathrm{L}$ & NO & Av & $\mathrm{NO}$ & $\mathrm{NO}$ & $\operatorname{Tr}$ \\
\hline GT2 & 290.2 & $\mathrm{~L}$ & $\mathrm{Ab}$ & $\mathrm{L}$ & Av & $\operatorname{Tr}$ & NO & $\mathrm{L}$ & $\mathrm{NO}$ & $\mathrm{L}$ & $\mathrm{NO}$ & NO & $\operatorname{Tr}$ \\
\hline GT4 & 290.4 & $\mathrm{~L}$ & $\mathrm{Av}$ & $\mathrm{Av}$ & $\mathrm{Av}$ & $\mathrm{NO}$ & $\mathrm{NO}$ & $\mathrm{L}$ & $\mathrm{Tr}$ & $\mathrm{L}$ & $\mathrm{NO}$ & $\mathrm{NO}$ & $\operatorname{Tr}$ \\
\hline GT5 & 290.7 & $\mathrm{~L}$ & $\mathrm{Ab}$ & $\mathrm{L}$ & $\mathrm{Av}$ & $\mathrm{NO}$ & $\mathrm{NO}$ & VL & VL & $\mathrm{Av}$ & $\mathrm{NO}$ & $\mathrm{NO}$ & $\operatorname{Tr}$ \\
\hline GT6 & 290.9 & $\mathrm{~L}$ & $\mathrm{Av}$ & $\mathrm{L}$ & $\mathrm{L}$ & NO & NO & $\operatorname{Tr}$ & VL & $\mathrm{Av}$ & $\mathrm{NO}$ & $\mathrm{NO}$ & $\mathrm{NO}$ \\
\hline GT7 & 292.6 & $\mathrm{~L}$ & $\mathrm{Av}$ & $\mathrm{L}$ & VL & NO & $\mathrm{NO}$ & $\mathrm{Tr}$ & L & $\mathrm{Av}$ & $\mathrm{NO}$ & $\mathrm{NO}$ & $\operatorname{Tr}$ \\
\hline GT8 & 303.3 & $\mathrm{~L}$ & $\mathrm{Ab}$ & VL & $\mathrm{Tr}$ & $\operatorname{Tr}$ & $\mathrm{NO}$ & $\mathrm{Tr}$ & $\mathrm{Tr}$ & VA & $\mathrm{Tr}$ & $\mathrm{NO}$ & $\operatorname{Tr}$ \\
\hline GT9 & 315.4 & $A v$ & $\mathrm{Av}$ & VL & NO & NO & $\mathrm{NO}$ & $\mathrm{L}$ & $\mathrm{Tr}$ & $\mathrm{Ab}$ & VP & $\operatorname{Tr}$ & $\mathrm{NO}$ \\
\hline GT10 & 321.4 & $A v$ & $\mathrm{Av}$ & VL & $\mathrm{NO}$ & $\mathrm{NO}$ & $\mathrm{NO}$ & $\mathrm{NO}$ & VL & $\mathrm{Ab}$ & VP & $\mathrm{NO}$ & $\operatorname{Tr}$ \\
\hline \multicolumn{14}{|c|}{ Sequence III } \\
\hline GT11 & 335.0 & $\mathrm{~L}$ & $\mathrm{Av}$ & $\mathrm{L}$ & $\operatorname{Tr}$ & $\mathrm{NO}$ & NO & VL & $\mathrm{L}$ & $\mathrm{Av}$ & $\mathrm{NO}$ & $\mathrm{NO}$ & $\operatorname{Tr}$ \\
\hline GT12 & 336,8 & $\mathrm{Av}$ & $\mathrm{L}$ & VL & $\operatorname{Tr}$ & $\mathrm{NO}$ & $\mathrm{NO}$ & VL & $\mathrm{NO}$ & VA & $\mathrm{NO}$ & $\mathrm{NO}$ & $\mathrm{NO}$ \\
\hline GT13 & 352.0 & $\mathrm{~L}$ & $\mathrm{Ab}$ & $\mathrm{L}$ & $\operatorname{Tr}$ & NO & $\mathrm{NO}$ & $\mathrm{Av}$ & VL & $\mathrm{Av}$ & $\mathrm{NO}$ & $\mathrm{NO}$ & $\operatorname{Tr}$ \\
\hline GT14 & 357.5 & $\mathrm{~L}$ & $\mathrm{Ab}$ & VL & $\operatorname{Tr}$ & $\operatorname{Tr}$ & $\mathrm{NO}$ & $\operatorname{Tr}$ & $\operatorname{Tr}$ & VA & VL & $\mathrm{NO}$ & $\mathrm{NO}$ \\
\hline GT15 & 385.0 & $\mathrm{~L}$ & $\mathrm{Ab}$ & VL & NO & $\mathrm{NO}$ & $\mathrm{NO}$ & $\mathrm{NO}$ & $\mathrm{NO}$ & VA & $\mathrm{NO}$ & $\mathrm{NO}$ & NO \\
\hline \multicolumn{14}{|c|}{ Sequence II } \\
\hline GT16 & 390.7 & $\mathrm{~L}$ & $\mathrm{Ab}$ & $\operatorname{Tr}$ & $\operatorname{Tr}$ & NO & NO & $\mathrm{NO}$ & $\operatorname{Tr}$ & $\operatorname{Tr}$ & $\mathrm{NO}$ & $\mathrm{NO}$ & $\mathrm{NO}$ \\
\hline GT17 & 398.5 & $\mathrm{~L}$ & Av & VL & $\mathrm{L}$ & $\mathrm{NO}$ & $\mathrm{NO}$ & $\operatorname{Tr}$ & $\operatorname{Tr}$ & $\mathrm{Ab}$ & $\mathrm{NO}$ & $\mathrm{L}$ & $\mathrm{NO}$ \\
\hline GT18 & 399.5 & $\mathrm{Ab}$ & $\mathrm{L}$ & VL & $\mathrm{Av}$ & $\mathrm{NO}$ & $\mathrm{NO}$ & VL & NO & $\mathrm{Av}$ & $\mathrm{NO}$ & $\mathrm{NO}$ & $\mathrm{NO}$ \\
\hline GT19 & 400.2 & $\mathrm{~L}$ & $\mathrm{Av}$ & VL & $\mathrm{L}$ & NO & NO & VL & $\operatorname{Tr}$ & $\mathrm{Ab}$ & VL & $\mathrm{NO}$ & $\mathrm{NO}$ \\
\hline GT20 & 401.5 & $\mathrm{~L}$ & $\mathrm{Ab}$ & $\mathrm{NO}$ & -- & -- & -- & -- & -- & -- & -- & $\mathrm{NO}$ & $\mathrm{NO}$ \\
\hline GT21 & 402.5 & $\mathrm{~L}$ & $\mathrm{Ab}$ & $\operatorname{Tr}$ & $\operatorname{Tr}$ & NO & $\mathrm{NO}$ & $\mathrm{NO}$ & NO & $\operatorname{Tr}$ & $\mathrm{NO}$ & $\operatorname{Tr}$ & $\operatorname{Tr}$ \\
\hline GT22 & 414.0 & $\mathrm{Ab}$ & $\mathrm{L}$ & $\operatorname{Tr}$ & $\operatorname{Tr}$ & No & $\mathrm{NO}$ & $\mathrm{NO}$ & $\mathrm{NO}$ & $\operatorname{Tr}$ & $\operatorname{Tr}$ & No & $\operatorname{Tr}$ \\
\hline GT23 & 417.5 & $\mathrm{NO}$ & VL & $\mathrm{NO}$ & -- & -- & -- & -- & -- & -- & -- & $\mathrm{NO}$ & $\mathrm{NO}$ \\
\hline
\end{tabular}


Tab. 3. Petrographic composition analysis for sample of disperse organic matter on the whole rock and organic concentrate, in reflected white light and fluorescence light. Legend: Depth (m); V- Vitrinite; I- Inetinite; L- Liptinite; Esp- Sporinite; Cut- Cutinite; Res- Resinite; Tel- Telalginite; Lam- Lamalginite; Mig- Migrabitumen; Lip- Liptodetrinite; MOM- Mineral Organic Matter; Bet- Betuminite; VA- Very abundant (>90\%); Ab- Abundant (90-70\%); Av- Average (69-40\%); L -Low (39-10\%); VL- Very low (<10\%); Tr- Trace (<6 ponts); NO- Not Observed.

\begin{tabular}{|c|c|c|c|c|c|c|c|c|c|c|c|c|c|}
\hline \multirow{3}{*}{\multicolumn{2}{|c|}{$\begin{array}{l}\text { Samples } \\
\text { reference }\end{array}$}} & \multicolumn{12}{|c|}{ Borehole 5-CA-81-RS } \\
\hline & & \multicolumn{10}{|c|}{ Structured Organic Matter } & \multirow{2}{*}{\multicolumn{2}{|c|}{$\begin{array}{l}\text { Unstructured } \\
\text { Organic Matter }\end{array}$}} \\
\hline & & \multicolumn{3}{|c|}{ Macerals Group } & \multicolumn{6}{|c|}{ Macerals Group of Liptinite } & \multirow[t]{2}{*}{ Mig } & & \\
\hline Sample & Depth & $\mathbf{V}$ & I & $\mathbf{L}$ & Esp & Cut & Res & Tel & Lam & Lip & & MOM & Bet \\
\hline \multicolumn{14}{|c|}{ Sequence IV } \\
\hline CA1 & 189.2 & $\operatorname{Tr}$ & $\mathrm{Va}$ & $\mathrm{NO}$ & -- & -- & -- & -- & -- & -- & -- & $\mathrm{NO}$ & $\mathrm{NO}$ \\
\hline CA2 & 199.3 & $\operatorname{Tr}$ & $\mathrm{Va}$ & $\operatorname{Tr}$ & $\operatorname{Tr}$ & $\mathrm{NO}$ & $\mathrm{NO}$ & $\mathrm{NO}$ & $\operatorname{Tr}$ & $\operatorname{Tr}$ & $\mathrm{NO}$ & $\mathrm{NO}$ & $\mathrm{NO}$ \\
\hline CA3 & 210.5 & $\mathrm{NO}$ & VA & VL & $\operatorname{Tr}$ & NO & NO & NO & $\operatorname{Tr}$ & VA & $\operatorname{Tr}$ & $\operatorname{Tr}$ & $\operatorname{Tr}$ \\
\hline CA4 & 220.4 & $\mathrm{~L}$ & $\mathrm{Ab}$ & $\mathrm{VL}$ & $A v$ & $\mathrm{NO}$ & $\mathrm{NO}$ & $\operatorname{Tr}$ & $\operatorname{Tr}$ & $\mathrm{Av}$ & $\mathrm{Tr}$ & $\mathrm{NO}$ & $\mathrm{NO}$ \\
\hline CA5 & 230.3 & $\mathrm{~L}$ & Av & $\mathrm{L}$ & $\mathrm{Av}$ & $\mathrm{NO}$ & $\mathrm{NO}$ & $\operatorname{Tr}$ & $\operatorname{Tr}$ & $\mathrm{Av}$ & $\mathrm{NO}$ & $\mathrm{NO}$ & $\mathrm{NO}$ \\
\hline CA6 & 234.7 & $\mathrm{~L}$ & Av & $\mathrm{L}$ & $A v$ & $\mathrm{NO}$ & $\mathrm{NO}$ & $\operatorname{Tr}$ & $\mathrm{NO}$ & $A v$ & $\mathrm{NO}$ & $\mathrm{NO}$ & $\mathrm{NO}$ \\
\hline CA7 & 240.8 & $\mathrm{~L}$ & Av & $\mathrm{L}$ & $A v$ & $\mathrm{NO}$ & $\mathrm{NO}$ & $\operatorname{Tr}$ & $\operatorname{Tr}$ & $\mathrm{Av}$ & $\mathrm{Tr}$ & $\mathrm{NO}$ & $\mathrm{NO}$ \\
\hline CA8 & 253.9 & $\mathrm{~L}$ & $A v$ & $\mathrm{~L}$ & $\mathrm{Av}$ & $\mathrm{NO}$ & $\mathrm{NO}$ & $\mathrm{Tr}$ & L & Av & $\mathrm{NO}$ & $\mathrm{NO}$ & $\mathrm{NO}$ \\
\hline CA9 & 258.5 & $\mathrm{~L}$ & $\mathrm{~L}$ & AV & $\mathrm{L}$ & NO & NO & VL & $\mathrm{L}$ & Av & $\mathrm{NO}$ & $\mathrm{VL}$ & $\mathrm{NO}$ \\
\hline \multicolumn{14}{|c|}{ Sequence III } \\
\hline CA10 & 277.6 & VL & $\mathrm{Av}$ & Av & $\mathrm{Av}$ & NO & NO & $\operatorname{Tr}$ & $\mathrm{L}$ & $\mathrm{L}$ & $\mathrm{NO}$ & $\mathrm{VL}$ & $\mathrm{NO}$ \\
\hline CA11 & 280.7 & $\mathrm{VL}$ & $\mathrm{Av}$ & $\mathrm{Av}$ & $\mathrm{NO}$ & $\mathrm{NO}$ & $\mathrm{NO}$ & $\operatorname{Tr}$ & $\mathrm{VL}$ & $\mathrm{VA}$ & $\mathrm{NO}$ & $\mathrm{NO}$ & $\mathrm{VL}$ \\
\hline CA12 & 286.6 & $\mathrm{~L}$ & $\mathrm{Ab}$ & VA & $\mathrm{NO}$ & $\mathrm{NO}$ & $\mathrm{NO}$ & $\mathrm{NO}$ & $\mathrm{Ab}$ & $\operatorname{Tr}$ & $\mathrm{NO}$ & $\mathrm{VL}$ & $\mathrm{NO}$ \\
\hline \multicolumn{14}{|c|}{ Sequence II } \\
\hline CA13 & 349.5 & $\mathrm{NO}$ & VA & $\mathrm{NO}$ & -- & -- & -- & -- & -- & -- & -- & $\mathrm{NO}$ & $\mathrm{NO}$ \\
\hline CA14 & 350.2 & $\mathrm{VL}$ & $\mathrm{Ab}$ & $\operatorname{Tr}$ & $\mathrm{NO}$ & $\mathrm{NO}$ & $\mathrm{NO}$ & $\mathrm{NO}$ & $\mathrm{NO}$ & $\operatorname{Tr}$ & $\mathrm{NO}$ & $\mathrm{VL}$ & $\mathrm{NO}$ \\
\hline CA15 & 352.5 & $\mathrm{VL}$ & $\mathrm{Ab}$ & $\operatorname{Tr}$ & $\operatorname{Tr}$ & $\mathrm{NO}$ & $\mathrm{Tr}$ & $\mathrm{NO}$ & $\mathrm{NO}$ & $\mathrm{NO}$ & $\mathrm{NO}$ & VL & $\operatorname{Tr}$ \\
\hline CA16 & 353.3 & VL & $\mathrm{VA}$ & $\operatorname{Tr}$ & $\mathrm{NO}$ & $\mathrm{NO}$ & $\mathrm{NO}$ & $\mathrm{NO}$ & $\mathrm{NO}$ & $\operatorname{Tr}$ & $\mathrm{NO}$ & $\operatorname{Tr}$ & $\mathrm{NO}$ \\
\hline CA17 & 355.3 & $\mathrm{VL}$ & VA & $\operatorname{Tr}$ & $\mathrm{NO}$ & $\operatorname{Tr}$ & $\mathrm{NO}$ & $\mathrm{NO}$ & $\mathrm{NO}$ & $\operatorname{Tr}$ & $\mathrm{NO}$ & $\operatorname{Tr}$ & $\mathrm{NO}$ \\
\hline CA18 & 358.4 & $\mathrm{VL}$ & $\mathrm{Ab}$ & $\operatorname{Tr}$ & $\mathrm{NO}$ & $\mathrm{NO}$ & $\mathrm{NO}$ & $\mathrm{NO}$ & $\mathrm{NO}$ & $\operatorname{Tr}$ & $\mathrm{NO}$ & $\mathrm{NO}$ & $\mathrm{NO}$ \\
\hline CA19 & 367.0 & $\mathrm{~L}$ & $\mathrm{Ab}$ & $\mathrm{NO}$ & -- & -- & -- & -- & -- & -- & -- & $\mathrm{NO}$ & $\mathrm{NO}$ \\
\hline CA20 & 371.8 & $\mathrm{VL}$ & VA & $\operatorname{Tr}$ & $\operatorname{Tr}$ & NO & $\operatorname{Tr}$ & $\mathrm{NO}$ & $\mathrm{NO}$ & $\operatorname{Tr}$ & $\mathrm{NO}$ & NO & $\mathrm{NO}$ \\
\hline CA21 & 377.7 & VL & VA & $\mathrm{NO}$ & -- & -- & -- & -- & -- & -- & -- & $\mathrm{NO}$ & $\mathrm{NO}$ \\
\hline CA22 & 381.3 & $\mathrm{~L}$ & $\mathrm{Ab}$ & $\mathrm{NO}$ & -- & -- & -- & -- & -- & -- & -- & $\mathrm{NO}$ & $\mathrm{NO}$ \\
\hline CA23 & 382.5 & $\mathrm{NO}$ & VA & $\mathrm{NO}$ & -- & -- & -- & -- & -- & -- & -- & $\mathrm{NO}$ & $\mathrm{NO}$ \\
\hline CA24 & 386.8 & $\operatorname{Tr}$ & VA & $\mathrm{NO}$ & -- & -- & -- & -- & -- & -- & -- & $\mathrm{NO}$ & $\mathrm{NO}$ \\
\hline CA25 & 399.6 & $\operatorname{Tr}$ & VA & $\operatorname{Tr}$ & $\mathrm{NO}$ & $\operatorname{Tr}$ & $\mathrm{NO}$ & $\mathrm{NO}$ & $\mathrm{NO}$ & $\operatorname{Tr}$ & $\mathrm{NO}$ & $\mathrm{NO}$ & $\mathrm{NO}$ \\
\hline CA26 & 413.6 & $\mathrm{~L}$ & $\mathrm{Ab}$ & $\operatorname{Tr}$ & $\mathrm{Tr}$ & $\mathrm{NO}$ & $\mathrm{NO}$ & $\mathrm{NO}$ & $\mathrm{NO}$ & $\mathrm{Tr}$ & $\mathrm{NO}$ & $\mathrm{NO}$ & $\mathrm{NO}$ \\
\hline CA27 & 416.6 & $\mathrm{NO}$ & VA & $\mathrm{NO}$ & -- & -- & -- & -- & -- & -- & -- & $\mathrm{VL}$ & $\mathrm{NO}$ \\
\hline
\end{tabular}


Tab. 4. Results from pyrolysis analysis in samples from borehole 2-TG-96-RS. Legend: ST-Sistems Tract; HST- Highstand Sistems Tract; TST- Transgressive Sistems Tract; LST - Lowstand Sistems Tract; Fm- Formation; TOC-Total Organic Carbon (\%); HI- Hidrogen Index (mg HC/g TOC); OI - Oxigen Index ( $\mathrm{mgCO}_{2} / \mathrm{g}$ TOC); S1 hidrocarbon already in rock (mg HC/g rock); S2 hidrocarbon from kerogen pyrolysis (mg HC/g rock).

\begin{tabular}{|c|c|c|c|c|c|c|c|c|}
\hline \multicolumn{9}{|c|}{ Borehole 2-TG-96-RS } \\
\hline ST & Sample & $\operatorname{Depth}(\mathrm{m})$ & $\mathrm{Fm}$ & TOC (\%) & HI & OI & S1 & $\mathbf{S} 2$ \\
\hline \multicolumn{9}{|c|}{ Sequence IV } \\
\hline \multirow{3}{*}{ TST } & TG9817 & 797.7 & Iratí & 2.2 & 10 & 11 & 0.13 & 0.22 \\
\hline & TG9818 & 800.9 & Iratí & 6.8 & 14 & 7 & 0.42 & 0.94 \\
\hline & TG9819 & 803.6 & Iratí & 0.73 & 15 & 22 & 0.09 & 0.11 \\
\hline \multicolumn{9}{|c|}{ Sequence III } \\
\hline \multirow{3}{*}{ HST } & 9613681.32 & 807.3 & Iratí & 1.15 & 71 & 43 & 1.09 & 0.82 \\
\hline & 9613683.58 & 809.2 & Iratí & 0.76 & 21 & 30 & 0.15 & 0.16 \\
\hline & TG9821 & 810.4 & Iratí & 1.41 & 148 & 19 & 0.56 & 2.08 \\
\hline \multicolumn{9}{|c|}{ Sequence II } \\
\hline \multirow{2}{*}{ HST } & TG9825 & 890.1 & Rio Bonito & 1.02 & 60 & 11 & 0.10 & 0.61 \\
\hline & TG9826 & 891.0 & Rio Bonito & 1.02 & 85 & 16 & 0.13 & 0.87 \\
\hline \multirow{16}{*}{ TST } & TG9827 & 891.5 & Rio Bonito & 1.22 & 117 & 17 & 0.21 & 1.43 \\
\hline & 9613692.59 & 893.7 & Rio Bonito & 1.01 & 124 & 41 & 0.26 & 1.25 \\
\hline & 9613693.92 & 894.2 & Rio Bonito & 1.19 & 145 & 16 & 0.23 & 1.73 \\
\hline & 9613694.76 & 895.9 & Rio Bonito & 1.27 & 98 & 20 & 0.13 & 1.24 \\
\hline & 9613696.33 & 897.4 & Rio Bonito & 18.67 & 267 & 6 & 2.4 & 49.9 \\
\hline & 9613697.17 & 897.9 & Rio Bonito & 16.63 & 155 & 14 & 2.4 & 25.7 \\
\hline & TG9828 & 910.0 & Rio Bonito & 11.68 & 173 & 3 & 0.89 & 20.16 \\
\hline & 9613699.84 & 926.9 & Rio Bonito & 55.15 & 62 & 7 & 6.0 & 34.0 \\
\hline & 9613700.76 & 927.6 & Rio Bonito & 34.0 & 103 & 29 & 5.0 & 35.0 \\
\hline & 9613701.51 & 940.2 & Rio Bonito & 27.88 & 32 & 7 & 3.0 & 9.0 \\
\hline & 9613702.33 & 940.8 & Rio Bonito & 26.61 & 26 & 53 & 4.0 & 7.0 \\
\hline & 9613703.17 & 943.1 & Rio Bonito & 9.53 & 139 & 2 & 1.02 & 13.22 \\
\hline & 9613704.52 & 952.6 & Rio Bonito & 5.63 & 60 & 9 & 0.25 & 3.35 \\
\hline & 9613705.84 & 953.5 & Rio Bonito & 2.89 & 25 & 6 & 0.14 & 0.72 \\
\hline & 9613706.68 & 958.4 & Rio Bonito & 1.12 & 35 & 13 & 0.07 & 0.39 \\
\hline & TG9833 & 959.2 & Rio Bonito & 6.32 & 38 & 117 & 0.16 & 2.39 \\
\hline \multirow{2}{*}{ LST } & TG9835 & 967.7 & Rio Bonito & 1.15 & 8 & 16 & 0.06 & 0.12 \\
\hline & TG9839 & 988.0 & Rio Bonito & 3.73 & 164 & 2 & 0.29 & 6.13 \\
\hline
\end{tabular}


Tab. 5. Results from pyrolysis analysis in samples from borehole 7-GT-10-RS. Legend: ST-Sistems Tract; HST- Highstand Sistems Tract; TST- Transgressive Sistems Tract; LST-Lowstand Sistems Tract; Fm- Formation; TOC-Total Organic Carbon (\%); HI- Hidrogen Index (mgHC/g TOC); OI- Oxigen Index (mgCO $2 / \mathrm{g}$ TOC); S1 hidrocarbon already in rock (mgHC/g rock); S2 hidrocarbon from kerogen pyrolysis (mgHC/g rock).

\begin{tabular}{|c|c|c|c|c|c|c|c|c|}
\hline \multicolumn{9}{|c|}{ Borehole 7-GT-10-RS } \\
\hline ST & Sample & $\operatorname{Depth}(\mathrm{m})$ & $\mathrm{Fm}$ & TOC ( $\%)$ & HI & OI & S1 & S2 \\
\hline \multicolumn{9}{|c|}{ Sequence IV } \\
\hline \multirow[t]{3}{*}{ HST } & GT2 & 279.0 & Teresina & 0.72 & 51 & 3 & 0.04 & 0.37 \\
\hline & GT3 & 289.5 & Serra Alta & 0.69 & 54 & 7 & 0.06 & 0.37 \\
\hline & GT5 & 290.7 & Serra Alta & 0.71 & 58 & 17 & 0.05 & 0.41 \\
\hline \multirow[t]{8}{*}{ TST } & GT6 & 290.9 & Serra Alta & 0.68 & 46 & 15 & 0.04 & 0.31 \\
\hline & GT7 & 292.6 & Serra Alta & 0.72 & 61 & 31 & 0.05 & 0.44 \\
\hline & 9612199.82 & 293.8 & Serra Alta & 0.69 & 42 & 10 & 0.03 & 0.29 \\
\hline & GT8 & 303.3 & Serra Alta & 0.77 & 29 & 6 & 0.04 & 0.22 \\
\hline & 9612202.32 & 312.6 & Serra Alta & 4.27 & 39 & 1 & 1.39 & 1.65 \\
\hline & GT9 & 315.4 & Serra Alta & 0.81 & 77 & 10 & 0.07 & 0.62 \\
\hline & 9612205.82 & 320.9 & Serra Alta & 6.95 & 19 & 7 & 1.48 & 1.32 \\
\hline & GT10 & 321.4 & Serra Alta & 2.21 & 12 & 19 & 0.63 & 0.26 \\
\hline \multicolumn{9}{|c|}{ Sequence III } \\
\hline HST & GT11 & 335.0 & Iratí & 0.68 & 31 & 19 & 0.05 & 0.21 \\
\hline \multicolumn{9}{|c|}{ Sequence II } \\
\hline \multirow[t]{3}{*}{ HST } & GT17 & 398.5 & Rio Bonito & 0.68 & 37 & 18 & 0.04 & 0.25 \\
\hline & GT18 & 399.5 & Rio Bonito & 0.71 & 34 & 18 & 0.03 & 0.24 \\
\hline & GT19 & 400.2 & Rio Bonito & 0.76 & 38 & 21 & 0.04 & 0.29 \\
\hline \multirow[t]{4}{*}{ TST } & GT20 & 401.5 & Rio Bonito & 2.17 & 8 & 30 & 0.05 & 0.17 \\
\hline & 96122221.35 & 403.5 & Rio Bonito & 0.70 & 4 & 20 & 0.03 & 0.03 \\
\hline & 96122224.86 & 415.5 & Rio Bonito & 5.09 & 13 & 4 & 0.07 & 0.67 \\
\hline & GT23 & 417.5 & Rio Bonito & 4.26 & 4 & 7 & 0.07 & 0.19 \\
\hline
\end{tabular}

The samples of the Sequence III are positioned in the base of the Iratí Formation that covers a large area of Paraná Basin, and contains facies, which could potentiality, generate hydrocarbons. In Sequence III, the data points indicated in the diagram (Fig. 4), have low values of hydrogen and oxygen indexes. When the petrographic data is included with the above information, kerogen Type IV is suggested. Exceptions occurs in samples CA-10 and CA-11 (Table 2), where the liptinite group contains similar concentrations of inertinite, making it difficult to classify the kerogen as either Type II or Type III. In the diagram of Sequence III (Fig. 4), the samples CA-10 (HI $=34 \mathrm{mg} \mathrm{HC} / \mathrm{g}$ TOC and OI $=20 \mathrm{mg}$ $\mathrm{CO}_{2} / \mathrm{g}$ TOC) and CA-11 (HI=69 mg HC/g TOC and $\mathrm{OI}=18 \mathrm{mg} \mathrm{CO} 2 / \mathrm{g}$ TOC; Table 6 ) are located close to the origin of the chart, making difficult to distinguish between kerogen Type III and Type IV. As indicated previously, the organic matter is considered Type III, containing a mixture of the organic components of Types II and IV. 
Tab. 6. Results from pyrolysis analysis in samples from borehole 5-CA-81-RS. Legend: ST-Sistems Tract; HST- Highstand Sistems Tract; TST- Transgressive Sistems Tract; LST-Lowstand Sistems Tract; Fm- Formation; TOC-Total Organic Carbon (\%); HI- Hidrogen Index (mg HC/g TOC); OI- Oxigen Index (mg CO $2 / g$ TOC); S1 hidrocarbon already in rock (mg HC/g rock); S2 hidrocarbon from kerogen pyrolysis (mg HC/g rock).

\section{Borehole 5-CA-81-RS}

\begin{tabular}{|c|c|c|c|c|c|c|c|c|}
\hline ST & Sample & $\operatorname{Depth}(\mathrm{m})$ & $\mathrm{Fm}$ & TOC (\%) & HI & OI & S1 & $\mathbf{S} 2$ \\
\hline \multicolumn{9}{|c|}{ Sequence IV } \\
\hline HST & CA5 & 230.3 & Serra Alta & 0.73 & 23 & 38 & 0.05 & 0.17 \\
\hline \multirow{3}{*}{ TST } & CA7 & 240.8 & Serra Alta & 0.70 & 26 & 26 & 0.06 & 0.18 \\
\hline & 9613622.77 & 257.4 & Iratí & 0.81 & 109 & 4 & 0.10 & 0.88 \\
\hline & CA9 & 258.5 & Iratí & 0.86 & 150 & 12 & 0.11 & 1.29 \\
\hline \multicolumn{9}{|c|}{ Sequence III } \\
\hline \multirow{3}{*}{ HST } & CA10 & 277.6 & Iratí & 0.83 & 34 & 20 & 0.10 & 0.28 \\
\hline & CA11 & 280.7 & Iratí & 1.10 & 69 & 18 & 0.10 & 0.76 \\
\hline & 9613626.51 & 283.8 & Palermo & 0.72 & 67 & 8 & 0.08 & 0.48 \\
\hline \multicolumn{9}{|c|}{ Sequencia II } \\
\hline HST & CA13 & 349.5 & Rio Bonito & 1.13 & 15 & 10 & 0.10 & 0.17 \\
\hline \multirow{6}{*}{ TST } & CA16 & 353.3 & Rio Bonito & 0.98 & 10 & 6 & 0.06 & 0.10 \\
\hline & 9613635.52 & 360.4 & Rio Bonito & 9.86 & 140 & 5 & 0.37 & 13.83 \\
\hline & CA20 & 371.8 & Rio Bonito & 23.85 & 46 & 29 & 1.0 & 8.0 \\
\hline & 9613640.99 & 375.0 & Rio Bonito & 0.99 & 36 & 22 & 0.06 & 0.36 \\
\hline & CA21 & 377.7 & Rio Bonito & 1.05 & 15 & 30 & 0.06 & 0.16 \\
\hline & CA22 & 381.3 & Rio Bonito & 2.78 & 15 & 6 & 0.09 & 0.42 \\
\hline \multirow[t]{5}{*}{ LST } & CA23 & 382.5 & Rio Bonito & 1.69 & 11 & 6 & 0.07 & 0.19 \\
\hline & 9613646.82 & 402.9 & Rio Bonito & 0.79 & 65 & 33 & 0.35 & 0.51 \\
\hline & 9613649.21 & 414.1 & Rio Bonito & 1.64 & 24 & 20 & 0.08 & 0.39 \\
\hline & CA27 & 416.4 & Rio Bonito & 1.36 & 21 & 19 & 0.12 & 0.29 \\
\hline & 9613651.65 & 416.6 & Rio Bonito & 1.92 & 23 & 6 & 0.06 & 0.44 \\
\hline
\end{tabular}

Sequence IV is similar to Sequence III containing low HI values, low OI values and a combination of kerogen Types III and IV (Tables 4, 5 and 6; Fig. 4). Petrographic evaluation confirms this analysis as shown in Tables 1,2 and 3. Exceptions to this general categorization are samples CA-9 and 9613622.77 (borehole 5-CA-81-RS), located in the top of the Iratí Formation. Both samples contain higher percentages of HI (between 100 and $150 \mathrm{mg} \mathrm{HC} / \mathrm{g}$ TOC; Table 6, Fig. 4). Although, the first sample has low values for HI and OI, the petrographic results for the sample CA-9 (Table 3), indicate a predominance of the liptinite group. In the other sample, petrographic investigation was not conducted due to the proximity of the previous sample (90 $\mathrm{cm})$. The kerogens of these samples are classified as type III, resulting from the mixture of organic kerogen constituents Types II and IV.

\section{Discussion}

In every sequence within the study area, a predominance of the terrestrial OM (vitrinite + inertinite) has been observed. This data demonstrates that in the area containing boreholes 2-TG-96-RS, 7-GT-10-RS and 5-CA-81-RS, the supply and preservation conditions were more favourable for the continental $\mathrm{OM}$ fragments. The inability to differentiate between the samples of the systems tracts is 
related to the predominance of terrestrial OM. As previously mentioned, some samples had a slightly different organic composition. The samples TG-9826, TG-9827 and TG-9828 (borehole 2- TG-96-RS), belonging to Sequence II (Rio Bonito Formation), are enriched in microsporinite.

\section{RESEARCH PAPER}

These samples are associated with the sedimentary interval of the Paraná Basin containing coal layers. The concentration of microsporinites in these samples resulted from vegetation that proliferated along the water body margins prior to the deposition of peat.
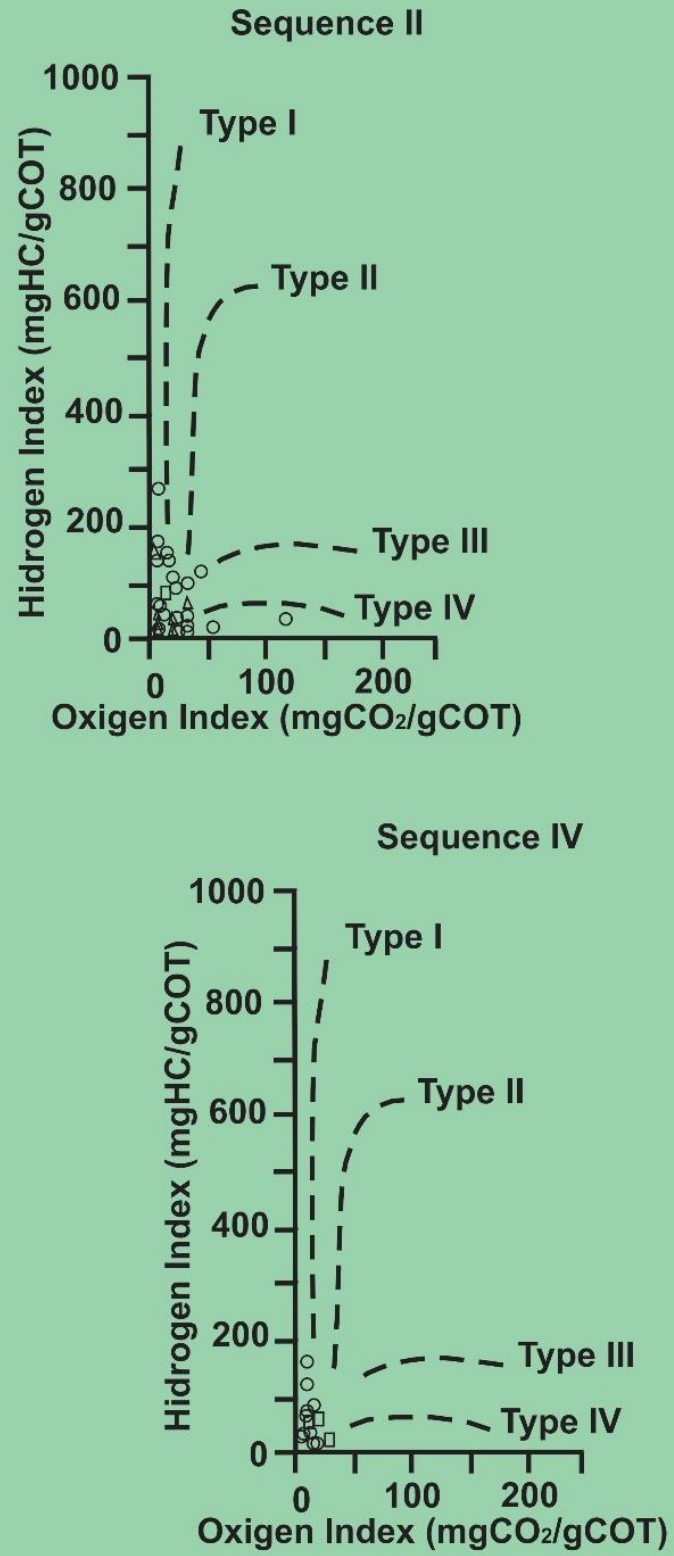

Sequence III

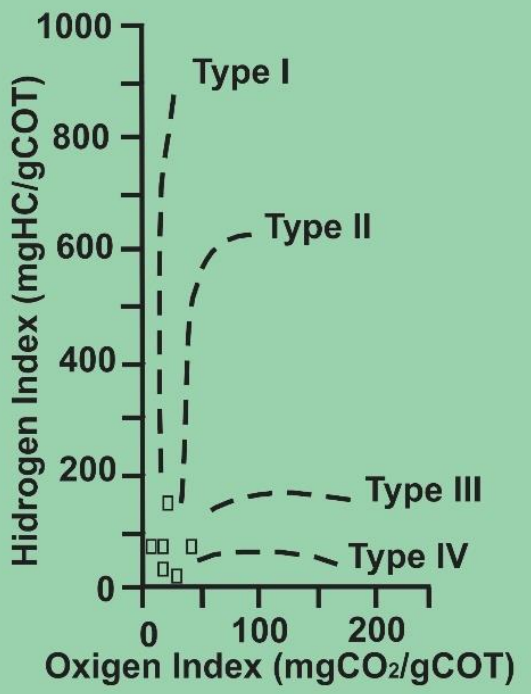

Highstand System Tract

O Trangressive System Tract

$\Delta$ Lowstand System Tract

Fig. 4. HI versus OI diagram based on Rock-Eval pyrolysis of whole rocks on samples Sequences II, III and IV. 
Of the entire sedimentary interval within the Paraná Basin, the shales of the Iratí Formation have more OM research studies related to oil exploration. The Iratí Formation in the Paraná Basin has been cited as an oil shale by several authors (such as Amaral, 1971; Schneider et. al., 1974; Burjack, 1984; Corrêa da Silva and Cornford, 1985; Araujo and Triguis, 1994). The Iratí shale is considered an oil source rock since it contains kerogen Types I and II.

Goulart and Jardim (1982), Cerqueira and Santos Neto (1986) observed that toward the west and southeast regions, the Irati Formation, shows a large reduction in $\mathrm{HI}$ values due to the influence of continental OM, identified by organic petrography (Fig. 5).

Burjack (1984), in a transmitted light microscopic study of sediments of the Iratí Formation, in the central and easterly regions of the Rio Grande do Sul State, recognized 3 different organic associations, informally called: sapropelic organopalinofacies, exinite-wood organopalinofacies and vitro-inertinite organopalinofacies. Burjack (1984), analyzing samples from borehole 7-GT-5-RS, next to the borehole 2TG-96-RS, determined that the Iratí Formation contains a mixture of organic matter consisting of melogen with a secondary component of amorphous B material. The samples analysis from borehole 7-GT-5-RS revealed a predominance of organopalinofacies, vitro-inertinite and melogen. This facies contains upper vegetable fragments, which could be correlated to the kerogen Types III or IV. Analysing samples from borehole 5-GA-80-RS, next to the borehole 5-CA-81-RS, Burjack (1984) observed an increase in the concentration of amorphous organic substances; however, the samples still contained high concentrations of melogen, with organopalinofacies and vitro-inertinite. According to Burjack (1984), and shown in a west-east crosssection, the melogen constituent increases in an easterly direction away from borehole 5-CA-81-RS.

The results of this study are similar and corroborates the results indicated by the mentioned authors, in regards to the type of the recognized kerogen. Based on the kerogen Type (III and IV) identified in the analyzed sequences, it can be deduced that the sedimentary rocks of this study do not have the potential for the generation of hydrocarbons. The lack of OM (typical of marine environments) in the studied sedimentary section should be a consequence of two factors: low productivity and poor preservation of marine organic matter (Parrish, 1995). In fact, the environment for the production and preservation of the $\mathrm{OM}$ in the study area should have been unfavourable because the area was proximal to the basin margin. The influx of continental freshwater, the shallow depth of water and the storm activity during the filling of the basin (Holz, 1997) should have acted as inhibiting factors for the production and preservation of organic material of marine organisms. The predominance of inertinite (the most oxidized $\mathrm{OM}$ ), vitrinite (terrestrial $\mathrm{OM}$ ) and the low concentrations of liptinite (alginites) indicate that the paleoenvironment was in fact not favourable to the production and preservation of $\mathrm{OM}$ supplied by marine productivity.

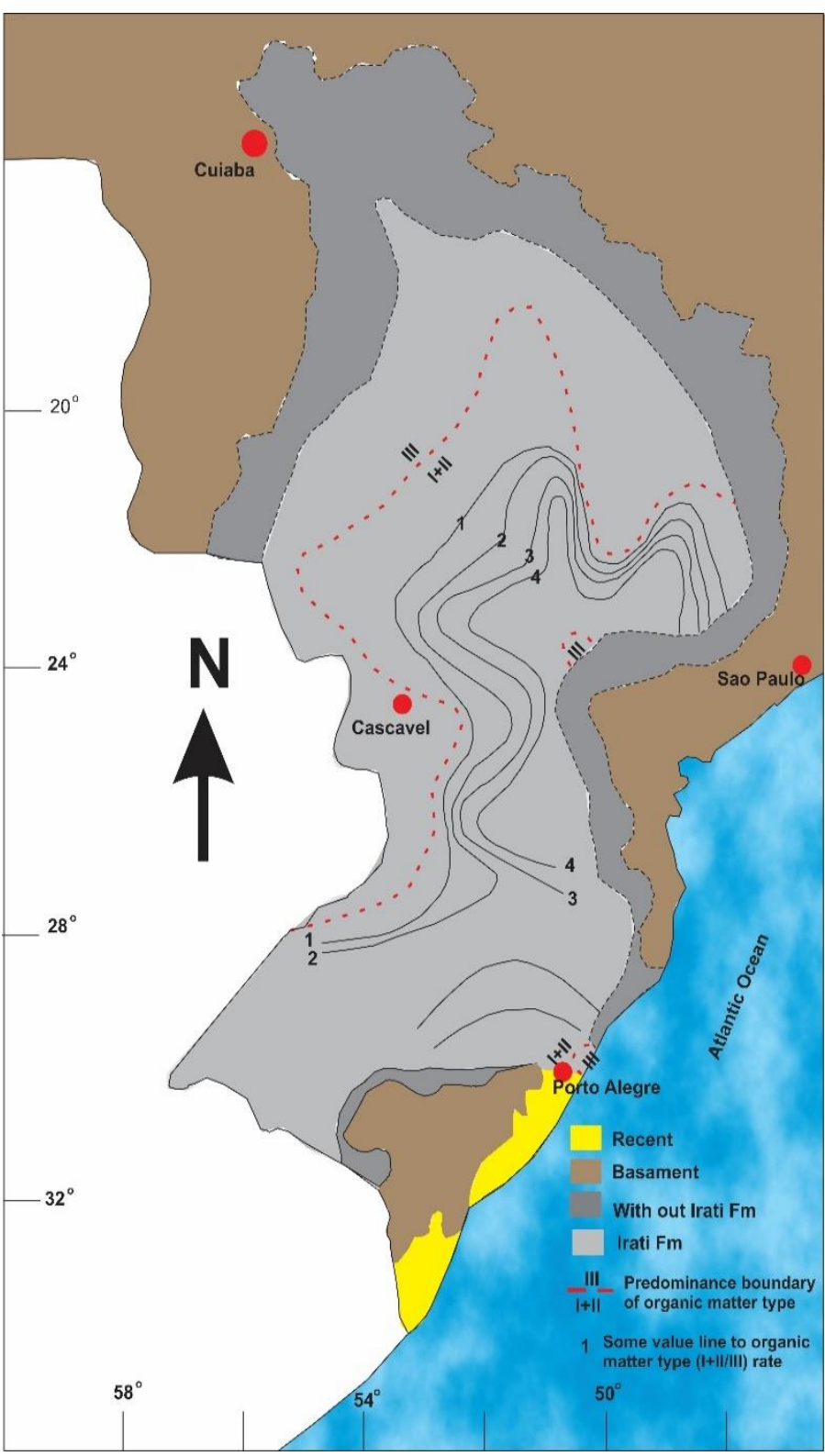

Fig. 5. Organic matter type distribution map on Iratí Formation, since I+II/III rate (modified from Goulart and Jardim, 1982).

\subsection{Organic Matter Maturation}

The thermal evolution of OM during diagenesis, catagenesis and metagenesis, modifies the physical and chemical properties of organic fragments. The variations in these properties are indicative of maturation (Tissot and Welte, 1978). The maturation of $\mathrm{OM}$ is controlled by temperature and time element of continuous burial, with the end product of this process being graphite (Teichmüller, 1975). 
According to Tissot and Welte (1978), shortly after burial (diagenesis), bacterial activity, partially consumes the organic biopolymers. The unconsumed fractions undergo polycondensation and insolubilization forming geopolymers with kerogen as the end product. A vitrinite reflectance of approximately $0.5 \%$ indicates the completion of this beginning phase of alteration. In the next phase (catagenesis) the continuing increase in temperature and pressure from burial, hydrocarbons will be generated by the thermal degradation of the kerogen, provided that the rock has sufficient organic matter. A vitrinite reflectance of about $2 \%$ marks the end of the second alteration phase (catagenesis), which produced oil and humic gas. Continual burial with the resulting increase in temperature and pressure leads to the final phase of the organic maturation process (metagensis). The source rocks during metagenesis contain only small concentrations of carbon and only gas is generated. A vitrinite reflectance of about $4 \%$ marks the end of this alteration phase after which mineral metamorphism occurs.

The maturation of OM can also be called, the thermal evolution of $\mathrm{OM}$, or organic matter metamorphism. The quantification of this process characterizes the thermal history of the sedimentary rock within the sedimentary basin.

Hoffmann and Jenkner (1932; in Teichmüller, 1975) observed that the reflectivity of coal vitrinite, increased with the quality ranking of the coals. McCartney (1952; in Teichmüller, 1975) quantified the above observations by using a photomultiplier to determine the vitrinite reflectance. Vitrinite reflectance measurements created an International Standard for the quality of coal or the degree of coalification. This technique was further used for the determination of the OM maturation in the siliciclastic rocks.

According to Bostick (1979), DOM solids occur only as a minor constituent in sedimentary rocks. DOM consists of diverse materials similar to the materials found in coals, but in different proportions than in normal coals. Consequently, DOM maturation chemically and physically approximates the coalification process. Bostick (1979) concludes that the vitrinite in the sediments, which has not undergone any secondary weathering processes, is the best particle for the evaluation of thermal history of rocks.

In accordance with Teichmüller (1987) the study of the organic content in the sediments is much more useful, in the determination of the thermal gradient of the sedimentary package rather than using other minerals. The $\mathrm{OM}$ is much more sensitive to heat variations than the pressure variations. Temperature related to the depth of burial, thermal gradient and termal conductivity is the critical factor controlling the chemical reactions in rocks. Beginning in the 70 's, vitrinite reflectance as a maturation parameter became widely used by oil companies in their exploration philosophies.

Although the vitrinite reflectance, in general, is the best parameter for the evaluation of thermal metamorphism of the DOM, it has some problems and limitations indicated below (Castaño and Sparks, 1974; Teichmüller, 1987; Robert, 1988; Senftle and Landis, 1991; Lo, 1992; Mukhopadhyay, 1994):

1- Above ground plant material, evolved in the Devonian, and therefore vitrinite cannot be found in the older rocks.

2- Statistically, a minimum of 30 to 50 measurements of vitrinite reflectance is necessary to draw a meaningful conclusion.

3- The vitrinite reflectance measurement made in whole-rock is more accurate than that carried out in organic concentrate, because the acid used to get the organic concentrate, causes some modification to the material;

4- The distinction between primary, secondary and oxidized vitrinite is not always clear, when the analysed particles are very small;

5- In very immature rocks, the $\mathrm{OM}$ is not useful in determining maturation;

6- Hydrogen enriched vitrinite lowers the vitrinite reflectance values, resulting from the incorporation of lipids in the biopolymers derived from degraded cuticle, algal or resin material;

7- The thermal conductivity and the capacity of heat retention are characteristics that change from rock to rock. Different physical and chemical reactions occur within the $\mathrm{OM}$, from rocks of different compositions, resulting in the reduction of the vitrinite reflectance values;

8- Bitumen impregnations or migrated oil retained in the vitrinite can also decrease vitrinite reflectance values.

As a result, the use of multiple parameters in the evaluation of the thermal history of a rock is always recommended (Teichmüller, 1987). According to Mukhopadhyay (1994), there are more than twenty parameters cited in the literature that can be used in the evaluation of the thermal evolution of OM; however, they all have their limitations. The cited physical parameters include: vitrinite reflectance, spore color index, thermal alteration index, concordant colour index and the evaluation of the intensity of fluorescence in spores and algal (maimum intensity) determined by microscopic examination. The cited chemicals parameters of maturation include but are not limited to: Tmax, sterane ratio normal C29 [205/(205 + $20 \mathrm{R})]$ and the ratio between diasterane and total C27 of

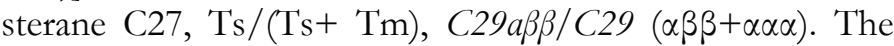
evaluation of the degree of maturation of the rocks in this study, used a combination of the physical parameter vitrinite reflectance - and the chemical parameter - Tmax.

According to Katz (1983), Espitalié et al. (1985), Peters (1986) and Wang (1989) the Tmax value will not be accurate when the generating potential (S2 peak) is lower than $0.2 \mathrm{mg}$ $\mathrm{HC} / \mathrm{g}$ rock. In this circumstance, it is advisable to disregard the Tmax values due to the sensitivity of the equipment. 
Some of the analysed rock samples (Table 4, 5 and 6) had S2 values lower than $0.2 \mathrm{mg} \mathrm{HC} / \mathrm{g}$ rock. Only sample 9612221.35 (Table 5) was disregarded, which had a Tmax value for S2 of $0.03 \mathrm{mgHC} / \mathrm{g}$ rock. All other samples that were below $0.2 \mathrm{mg} \mathrm{HC} / \mathrm{g}$ rock were kept do to the correlation with the vitrinite reflectance measurements. The analysis results have been summarized in Table 7 and Fig. 6.

In the southwestern extremity of the area (borehole 5CA-81-RS), the Tmax values varied between $332^{\circ} \mathrm{C}$ and $514^{\circ} \mathrm{C}$ having a random vitrinite reflectance $(\mathrm{Rr})$ varying between $0.40 \%$ and $1.9 \%$. In the sedimentary column from $190 \mathrm{~m}$ to $340 \mathrm{~m}$ of borehole 5-CA-81-RS, the OM for thermal evolution (diagenesis) is immature as indicated by the Rr and Tmax measurements. The oil generation window was encountered between $350 \mathrm{~m}$ and $417 \mathrm{~m}$, in the Rio Bonito Formation. The values of Tmax and $\mathrm{Rr}$ measured at a depth of $380 \mathrm{~m}$ suggest a higher maturation stage for the kerogen (sterile and dry gas, metagenesis).

In borehole 7-GT-10-RS the measured values of Tmax ranged from $411{ }^{\circ} \mathrm{C}$ to $526{ }^{\circ} \mathrm{C}$ and the measured $\mathrm{Rr}$ values ranged from $0.35 \%$ to $0.80 \%$. The immature stage (diagenesis) for OM was found from $250 \mathrm{~m}$ and $390 \mathrm{~m}$ based on the values of Tmax; however, the results from vitrinite reflectance measurements place the interval between immature and mature (diagenesis and catagenesis).

From $390 \mathrm{~m}$ until the end of the borehole the Rr suggests that the rocks reached the catagenesis stage, in Rio Bonito Formation, at the beginning of the oil generation window. The Tmax, for the same interval, varied from immature to mature.

In the northeast extremity of the study area (borehole 2TG-96-RS), Tmax measurements ranged from $432{ }^{\circ} \mathrm{C}$ to 523 ${ }^{\circ} \mathrm{C}$, and Rr values varied from $0.35 \%$ to $3.7 \%$. The Rr of the vitrinite indicates that the rock in the upper portion of the well (above $890 \mathrm{~m}$ ) is immature with exception of an anomaly that will be discussed later. Below $890 \mathrm{~m}$ (Rio Bonito Formation), both the Tmax and Rr parameters, indicate the occurrence of catagenetic phase of maturation. The anomalous portion of the well was found between $744 \mathrm{~m}$ and $810 \mathrm{~m}$. In this interval, the OM begins in the catagenesis phase reaching the maximum metagenesis phase at $794 \mathrm{~m}$. This anomalous rapid increase in both the Tmax and $\mathrm{Rr}$ values (Fig. 6) is the result of a local nearby igneous intrusion.

A similar igneous intrusion effect can be observed in borehole 5-CA-81-RS at the depth of $383 \mathrm{~m}$. Below this depth the measured values for Tmax and $\mathrm{Rr}$ equate with similar lower values in offset wells.

A second anomaly $(416 \mathrm{~m})$ showing very high measurements of Tmax and $\mathrm{Rr}$ is found at the bottom of the borehole. This increase in the values Tmax and Rr suggest an intrusive igneous body a few meters below the total depth of the well. An anomalous increase in maturation values was also observed near the bottom of borehole 7-GT-10-RS.
From the measurements of Tmax and $\mathrm{Rr}$, the top of the oil generation window is found in the Rio Bonito Formation. Above this formation, the sedimentary section is immature, with the exception of a few locals, which were proximal to local igneous intrusive rocks. These igneous intrusive rocks were small in aerial extent and did not contribute to the oil generating capacity of the basin.

\subsection{Organic Matter in the Sequence Stratigraphic Framework}

In boreholes 5-CA-81-RS, 7-GT-10-RS and 2-TG-96-RS, Sequences II, III and IV, according to Holz (1997), and Sequence SD3, according to Edgeton (1997), were analysed. Based on the data from Araújo and Triguis (1994) and Triguis et aI. (1996), the lower boundary of Sequence SD3 of Edgeton (1997), which is the top of the carbonates, was correlated to the base of Sequence II of Holz (1997) stratigraphic nomenclature.

The stratigraphic terminology used in this study was that of the SD3 Sequence of Edgeton (1997). The results of the petrography study are shown in Tables 1, 2, 3, and the pyrolysis results are shown in Tables 4, 5 and 6. An integration of both studies for each of the above sequences is summarized in Fig. 7.

\subsubsection{Sequence II}

In boreholes 5-CA-81-RS and 2-TG-96-RS the TOC values are low $(<1 \%)$, ignoring the exception of coaly shale associated with coal seams. The $\mathrm{HI}$ is generally $<100 \mathrm{mg}$ $\mathrm{HC} / \mathrm{g}$ TOC with the maximum value of $267 \mathrm{mg} \mathrm{HC} / \mathrm{g}$ TOC observed in borehole 2-TG-96-RS. In this stratigraphic sequence, the largest concentrations of organic material belong to the inertinite group, which can be considered on average very abundant. The vitrinite group was classified as very little to little and the liptinite group has only modest (very little to trace) concentrations. Some Botryococcus and rare Acritarchs were found in the transgressive system tract (TST). In general, the size of organic particles does not exceed $60 \mu \mathrm{m}$.

The highest concentrations of TOC values from Sequence II, was observed in the Transgressive System Tract (TST) with lesser values in the Lowstand System Tract (LST) and Highstand System Tract (HST). The value of HI in this sequence follows the same TOC pattern for borehole 2-TG96-RS.

Borehole 5-CA-81-RS has a similar but less obvious relationship. Pasley et al. (1993) in the San Juan Basin of New Mexico also observed this pattern of high concentrations of TOC in the Transgressive Highstand Tract.

This pattern is also found in the vitrinite group with greater concentrations in the TST than the LST and HST as observed in borehole 5-CA-81-RS and 2-TG-96-RS and, in a lesser extent, in the borehole 7-GT-10-RS. 
Tab. 7. Tmax $\left({ }^{\circ} \mathrm{C}\right)$ and vitrinite reflectance $(\operatorname{Rr} \%)$ data in the boreholes studies.

\begin{tabular}{|c|c|c|c|c|c|c|c|c|}
\hline \multicolumn{3}{|c|}{ Borehole 5-CA-81-RS } & \multicolumn{3}{|c|}{ Borehole 7-GT-10-RS } & \multicolumn{3}{|c|}{ Borehole 2-TG-96-RS } \\
\hline Depth & Tmax & $\mathbf{R r}$ & Depth & $T \max$ & $\mathbf{R r}$ & Depth & $T \max$ & $\mathbf{R r}$ \\
\hline \multicolumn{3}{|c|}{ Teresina Formation } & \multicolumn{3}{|c|}{ Teresina Formation } & \multicolumn{3}{|c|}{ Teresina Formation } \\
\hline 199.3 & -- & $0.45-0.50$ & 255.9 & -- & $0.40-0.50$ & 691.6 & -- & $0.35-0.40$ \\
\hline 220.4 & -- & $0.45-0.50$ & 279.0 & 429 & -- & 706.4 & -- & $0.40-0.45$ \\
\hline \multicolumn{3}{|c|}{ Serra Alta Formation } & 289.5 & 427 & $0.35-0.45$ & \multicolumn{3}{|c|}{ Serra Alta Formation } \\
\hline 230.3 & 426 & $0.55-0.60$ & \multicolumn{3}{|c|}{ Serra Alta Formation } & 720,1 & -- & $0.53-0.54$ \\
\hline 234.7 & -- & $0.45-0.50$ & 290.2 & -- & $0.40-0.50$ & 723,5 & -- & $1.0-1.3$ \\
\hline 240.8 & 426 & $0.55-0.60$ & 290.4 & -- & $0.40-0.50$ & 744.0 & -- & $1.25-1.35$ \\
\hline 253.9 & -- & $0.50-0.55$ & 290.7 & 431 & $0.45-0.50$ & \multicolumn{3}{|c|}{ Iratí Formation } \\
\hline 257.4 & 440 & -- & 290.9 & 429 & $0.45-0.50$ & 794.1 & -- & $3.6-3.7$ \\
\hline 258.5 & 435 & $0.40-0.45$ & 292.6 & 436 & $0.50-0.55$ & 797.7 & 476 & $1.5-1.9$ \\
\hline \multicolumn{3}{|c|}{ Iratí Formation } & 293.8 & 428 & -- & 800.9 & 523 & $1.7-1.9$ \\
\hline 277.6 & 421 & $0.40-0.45$ & 303.3 & 432 & $0.55-0.60$ & 803.6 & -- & $1.4-1.6$ \\
\hline 280.7 & 428 & $0.40-0.50$ & \multicolumn{3}{|c|}{ Iratí Formation } & 807.3 & 429 & -- \\
\hline \multicolumn{3}{|c|}{ Palermo Formation } & 312.6 & 438 & -- & 809.2 & 432 & -- \\
\hline 283.8 & 433 & -- & 315.4 & 436 & $0.50-0.65$ & 810.4 & 441 & $0.50-0.60$ \\
\hline 286.7 & -- & $0.40-0.45$ & 321.4 & 411 & $0.50-0.60$ & 811.6 & 447 & -- \\
\hline \multicolumn{3}{|c|}{ Rio Bonito Formation } & 335.0 & 422 & $0.55-0.60$ & 816.5 & 435 & $0.50-0.55$ \\
\hline 349.5 & 450 & -- & \multicolumn{3}{|c|}{ Palermo Formation } & \multicolumn{3}{|c|}{ Palermo Formation } \\
\hline 350.2 & -- & $0.40-0.45$ & 336.8 & -- & $0.40-0.50$ & 865.1 & 440 & -- \\
\hline 352.5 & -- & $0.45-0.50$ & 352.0 & -- & $0.50-0.60$ & \multicolumn{3}{|c|}{ Rio Bonito Formation } \\
\hline 353.3 & 446 & $0.45-0.50$ & 357.5 & -- & $0.45-0.55$ & 890.1 & 445 & $0.40-0.50$ \\
\hline 355.3 & -- & $0.45-0.50$ & \multicolumn{3}{|c|}{ Rio Bonito Formation } & 891.0 & 443 & $0.75-0.85$ \\
\hline 360.4 & 440 & -- & 385.0 & -- & $0.50-0.55$ & 891.3 & 435 & -- \\
\hline 367.0 & -- & $0.70-0.75$ & 390.7 & -- & $0.55-0.65$ & 891.5 & 441 & $0.50-0.60$ \\
\hline 371.8 & 449 & $0.55-0.60$ & 398.5 & 438 & $0.55-0.65$ & 893.7 & 440 & -- \\
\hline 375.0 & 445 & -- & 399.5 & 438 & $0.70-0.80$ & 894.2 & 443 & -- \\
\hline 377.7 & 474 & $0.45-0.55$ & 400.2 & 439 & $0.50-0.60$ & 895.9 & 440 & -- \\
\hline 381.3 & 514 & 1.8-1.9 & 401.5 & 490 & 1.6-1.7 & 896.7 & 452 & -- \\
\hline 382.5 & 497 & -- & 402.5 & -- & $0.55-0.65$ & 897.4 & 435 & $0.80-0.85$ \\
\hline 386.8 & -- & $2.8-3.0$ & 414.0 & -- & $0.50-0.60$ & 897.9 & 438 & $0.75-0.85$ \\
\hline 399.6 & -- & $0.52-0.55$ & 415.5 & 515 & -- & 909.0 & 458 & -- \\
\hline 402.9 & 332 & -- & 417.5 & 526 & -- & 910.0 & 441 & $0.80-0.90$ \\
\hline 413.6 & -- & $0.65-0.70$ & & & & 926.9 & 450 & $0.80-0.85$ \\
\hline 414.1 & 459 & -- & & & & 927.6 & 453 & $0.80-0.85$ \\
\hline 416.4 & 474 & -- & & & & 940.2 & 454 & $0.95-1.05$ \\
\hline 416.6 & 500 & $1.25-1.30$ & & & & 940.8 & 458 & $0.90-1.0$ \\
\hline & & & & & & 941.5 & 440 & -- \\
\hline & & & & & & 943.1 & 452 & -- \\
\hline & & & & & & 952.6 & 459 & $1.05-1.15$ \\
\hline & & & & & & 953.5 & 468 & -- \\
\hline & & & & & & 958.4 & 464 & -- \\
\hline & & & & & & 959.2 & 470 & $0.85-0.90$ \\
\hline & & & & & & 967.7 & 460 & $0.90-0.95$ \\
\hline & & & & & & 980.3 & -- & $0.65-0.75$ \\
\hline & & & & & & 985.1 & -- & 1.0 \\
\hline & & & & & & 988.0 & 464 & $0.70-0.75$ \\
\hline & & & & & & 991.4 & 452 & $0.70-0.75$ \\
\hline & & & & & & 992.3 & 449 & -- \\
\hline & & & & & & 993.3 & 444 & -- \\
\hline & & & & & & 994.0 & 447 & -- \\
\hline & & & & & & 996.0 & 444 & -- \\
\hline & & & & & & 999.1 & 448 & $0.80-0.90$ \\
\hline
\end{tabular}




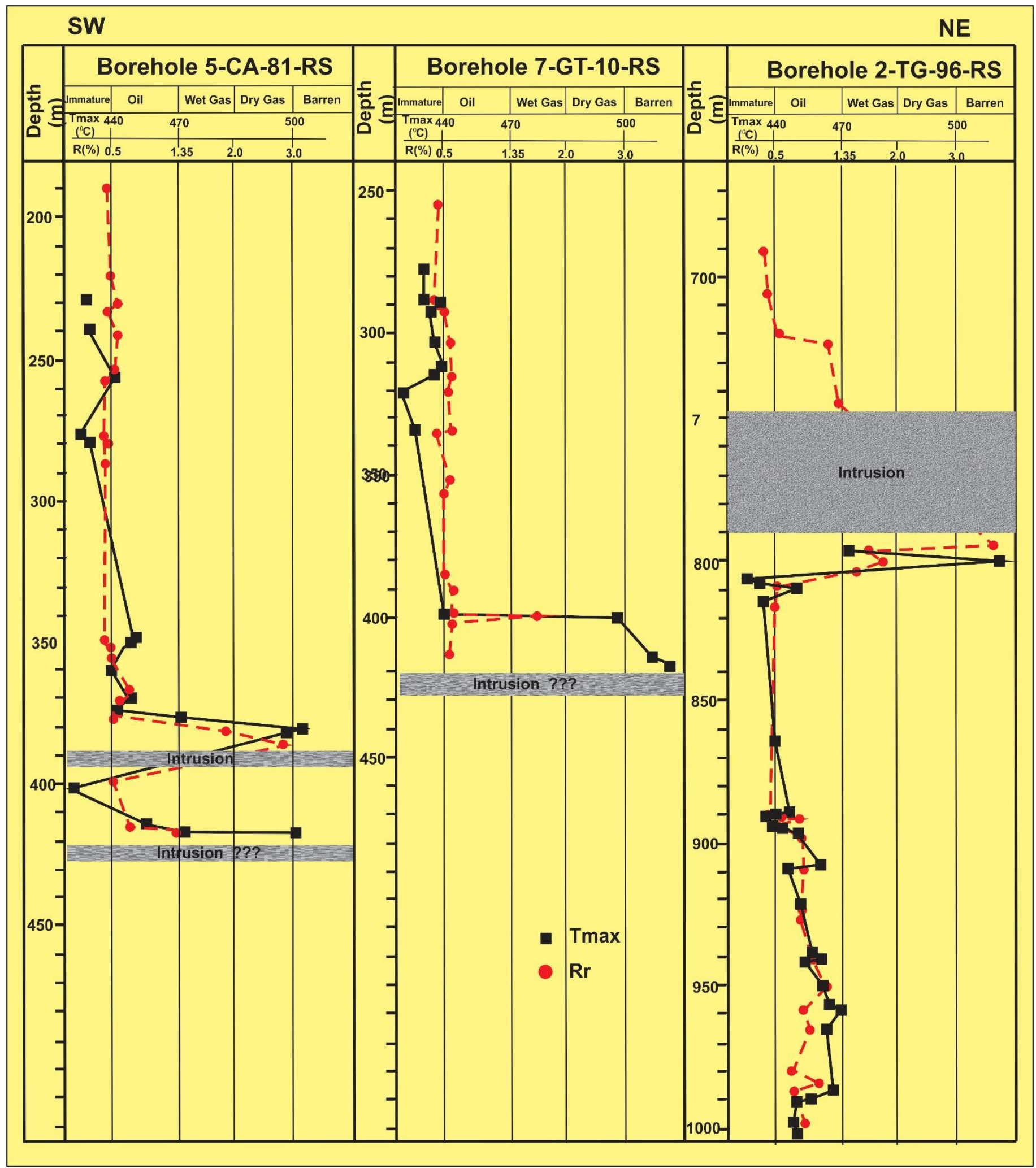

Fig. 6. Maturation of the sedimentary rocks package in Tmax and vitrinite reflectance $(\operatorname{Rr} \%)$. 
The inertinite group has a subtle opposite pattern in the three boreholes, with the lowest concentrations in the TST and the highest concentrations in HST and LST. The liptinite group also follows this opposite pattern with the exception in the 5-CA-81-RS borehole and the 2-TG-96-RS borehole where no distinction in concentration amounts could be made at the boundary between the LST and TST. In borehole 7-GT-10-RS, it was not possible to establish, any distinction using the liptinite group between the systems tracts for System II. Nevertheless, in this borehole, the liptinite group concentration increases in the TST at the maximum flooding surface (MFS). This concentration at the MFS was also observed in the borehole 2-TG-96-RS.

\subsubsection{Sequence III}

This sequence has a silty-sandstone lithology in two of the three analysed boreholes, causing difficulty in the interpretation and correlation of the data. Consequently, only borehole 7-GT-10-RS was analysed. The TOC log (Fig. 7) indicates no characteristics which allows the separation of the TST from the HST, due to the very low TOC $(<0.5 \%)$ values.

Due to the low abundance of the macerals group, the systems tracts of sequence III; show only an apparent but non-conclusive distribution pattern. Only one sample contained OM of the macerals group in the Shelf Margin System Tract (SMST) and in the TST.

Similar low concentrations of the vitrinite group prevented characterization of the three systems tracts. The inertinite group did indicate greater concentrations in the TST in relation to the HST. The liptinite group concentrations were higher in the HST than in the TST and TSMP. The liptinite group log (Fig. 7) shows a subtle correlation with largest abundances in the HST rather than the TST. In addition, organic particle size could not be used to distinguish differences between the systems tracts of this sequence. Botryococcus and some occurrences of Acritarchs predominately occurred only in the HST.

Higher TOC values and low HI values define the boundary between sequence III and IV (BS 4). This boundary could not be distinguished using the other measured parameters (vitrinite, inertinite, liptinite and particle size).

\subsubsection{Sequence IV}

In this sequence, the TST has high concentrations of TOC values and vitrinite than the HST. Conversely, the inertinite group showed greater concentrations in the HST than the TST. The trends for the HI and liptinite group concentrations were not definitive in the different boreholes.

In borehole 5-CA-81-RS the TST shows higher liptinite and $\mathrm{HI}$ concentrations than in the HST, with the opposite trend in borehole 7-GT-10-RS. The maximum flooding surface four (MFS 4) can be characterised by an increase in all measured parameters except particle size. Exceptions were found in borehole 7-GT-10-RS with a decrease of the vitrinite group at this event and in borehole 2-TG-96-RS with a decrease in TOC. This surface can be observed, in the materials concentrations log (Fig. 7) of borehole 2-TG-96RS, showing large concentrations of the measured parameters. Botryococcus was the main algal fossil observed; however, some Acritarchs were observed in the TST and HST next to the maximum flooding surface.

The TST of Sequences II and IV can be characterized by high concentrations of $\mathrm{HI}$ and the liptinite group and by low concentrations of the vitrinite group. Stach et al. (1975) and Taylor et al. (1998), in their studies of the materials group found, that liptinite shows higher amounts of " $\mathrm{H}$ " and inertinite group shows a smaller relative concentration of " $\mathrm{H}$. Consequently, the increase in the concentration of liptinite and vitrinite would imply an increase in the values of HI. In sequence IV, borehole 7-GT-10-RS, had the lowest concentrations of $\mathrm{HI}$ in the TST related to the reduction of the concentration of the liptinite group.

\subsection{Reconstruction the paleo-physiography of the area}

Holz (1997), reconstructing the paleo-physiography of the area, considered that Sequence II (bottom interval, LST) of the Rio Bonito Formation was deposited in fluvial-deltaic conditions and the upper part of this Formation was deposited in coastal plain environment (centre and top interval, TST and HST). The presence of coal zones and the predominance of terrestrial OM would also suggest a continental origin or depositional environment for this formation.

The presence of some Acritarchs, organisms typical of a saline environment in the LST, indicate the first marine incursion into the continental deposits of this Rio Bonito Formation. In the studied area, the sedimentary rocks lack the physical characteristics typical of marine deposits. However, the presence of the marine organisms (Acritarchs) indicate that short lived transgressions and regressions from storms tides occurred, which did not alter the overall sedimentary fabric of the formation.

In the Sequence III (Palermo Formation), according to Holz (1997), the sedimentary rocks were deposited in a tidal plain and bioturbated coastal plain environment, dominated by storms. Although this formation contains marine facies, nearby continental deposition strongly influenced the type and preservation of the OM. Continual tidal changes coupled with frequent storms prevented water stratification and caused reworking of deposited OM material. Coupled with bioturbation indicative of deep oxidizing conditions prevented the preservation of the deposited organic material. These conclusions are based on the low TOC concentrations $(<0,8 \%$, Tables 4,5 and 6$)$ and predominance of the inertinite (Tables 1, 2 and 3). 
sw

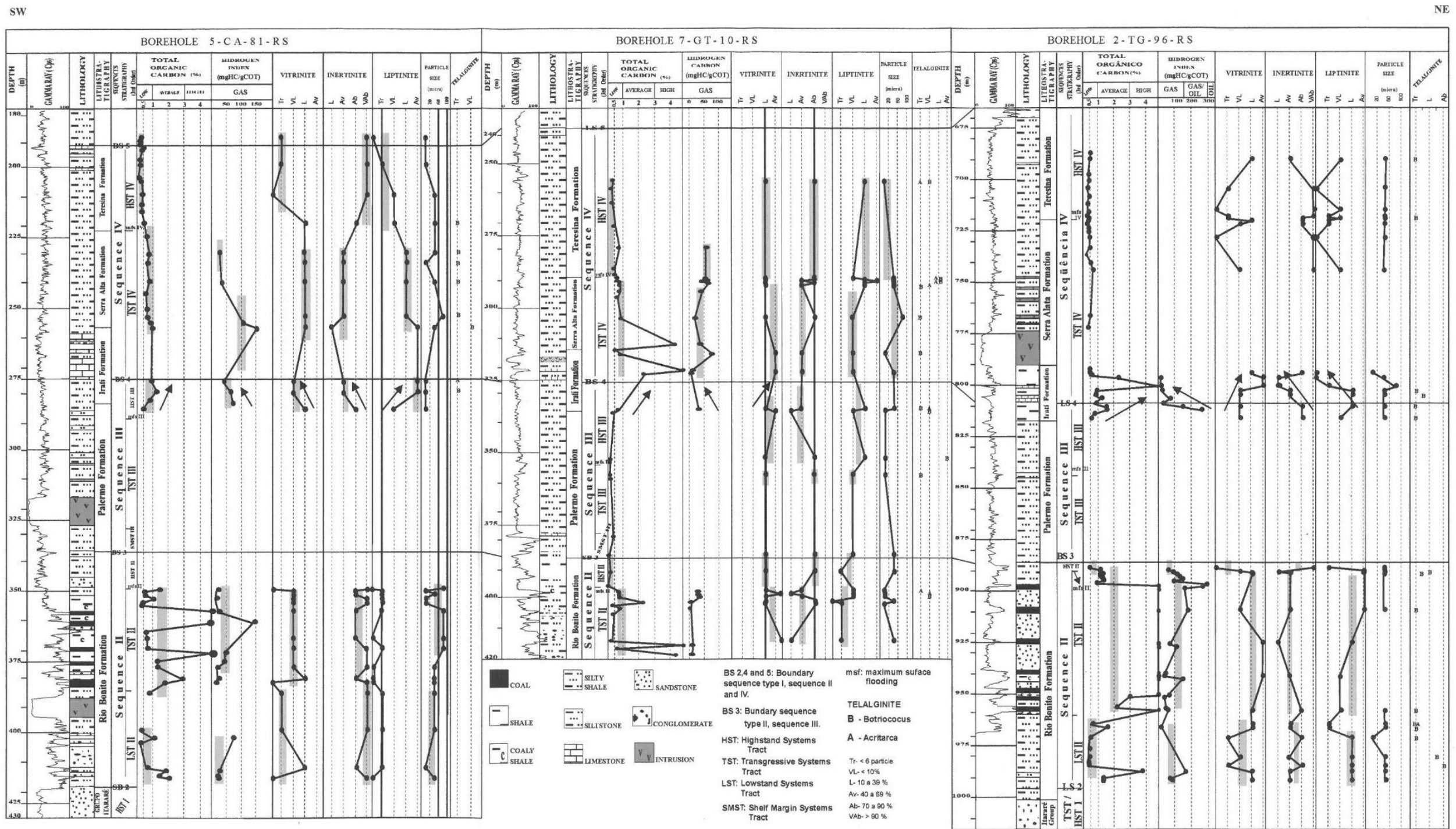

Fig. 7. Organic and stratigraphic parameters cross section. 
The boundary between Sequence III and Sequence IV (Iratí Formation) was deposited in a more distal restricted marine environment with little influence from storm waves. The resulting deep dysoxic conditions (Holz, 1997) reflected in sedimentary deposits, provided an increase in preserved TOC and OM concentrations. The restricted marine facies resulted in an increased preservation of the liptinite group in the HST 3 (boreholes 5-CA81-RS and 2-TG-96-RS). The marine facies associated with dysoxic conditions preserved the Acritarchs, algal fossils, but only few specimens were found. The change to a marine environment resulted in an increase of liptinite group; the dominant OM remained terrestrial.

Sequence IV, TST4 (Serra Alta Formation) and HST4 (Teresina Formation) were respectively deposited in transgressive and regressive marine shelf environments (Schneider et al., 1974; Milani et al., 2007). Despite the marine conditions during the deposition, only small amounts of $\mathrm{OM}$ were preserved, as evidenced by the low TOC values $(<1 \%$; Tables 4, 5 and 6). The reduction in the vitrinite group concentrations coupled with an increase in the inertinite group concentrations for TST4 and for the HST4 (boreholes 5-CA-81-RS and 7-GT-10-RS), suggests an increase in oxic conditions for the two sequences. Acritarchs fossils show the effects of degradation typical of a marine environment. A similar observation was also made by Schneider et al. (1974) and Milani et al. (2007).

Laterally, changes in oxidation intensity can be also observed in Sequence IV. In borehole 5-CA-10-RS, the variation of the vitrinite, inertinite and liptinite materials groups concentrations in the TST 4 from TSMA 4 indicates a wide range of values. In borehole 7-GT-10-RS, the vitrinite and inertinite materials groups also reach small percentages, with only the liptinite group showing a moderate increase in concentration. This suggests that laterally the depositional environment did not remain constant but went through several changes of water depth.

The DOM variations that can be recognized in continental shelf sediments are largely the result of the changing supply of terrigenous sediments (Pasley et al., 1993), as well as the distance of the OM deposition site from the shoreline (distal or proximal). In the study area, the DOM concentrations varied both laterally and vertically (Fig. 7). These variations in concentrations are associated with facies modifications from paleoclimate changes, according to $\mathrm{Holz}$ (1997), which increased or decreased the sedimentary supply to the basin. Although, climatic and facies changes did occur, these changes were not reflected in the organic content of the sedimentary rocks.

The terrestrial OM (vitrinite + inertinite) was a predominant component in all the sedimentary sequences, including at the marine environments. The small occurrences of the Acritarchs, algal fossils, in sequences III and IV, along with other liptinite materials resulted from degradation in a predominantly oxic environment (indicated by pyrolysis results).

Water is the main transporting agent of $\mathrm{OM}$ with wind a secondary mode of conveyance (Larsen and Chilingar, 1983). The smallest organic particles mainly of the liptinite group, which has the largest surface area, are spread more evenly throughout the environment; however, these particles are the most susceptible to the efficiency of degradation agents. Consequently, during long transport times an increase in the inertinite/vitrinite ratio indicates chemical degradation (oxidation).

In many lacustrine, fluvial, deltaic or shallow marine deposits, vitrinite is the most abundant group of terrigenous organic constituent, however, in most open marine deposits, inertinite predominates (Littke et al., 1997).

According to Littke (1993), the liptinite group has lower densities than the vitrinite and inertinite groups. Consequently, liptinite fragments remain in the transporter agent (water and wind) longer than the other groups and undergo more degradation. In addition, being richer in " $\mathrm{H}$ ", liptinites are more easily destroyed by weathering. Consequently, in marine deposits with deep oxic conditions, vitrinite and inertinite groups (terrestrial origin) remain the most common organic particle rather than materials of liptinite group (alginite) of marine origin.

Conversely, the predominance of terrestrial OM must also be associated with the proximity to the shoreline. The organic fragments consisting of cellulose and degraded resistant lignin, that constitutes the vitrinite and inertinite, would still be degraded when exposed to long periods of transport.

During regressive events (shoreline progradation), the sediments and $\mathrm{OM}$ are deposited in strand plain, delta and prodelta environments. Consequently, these materials are partially redistributed along the shelf by longshore currents and storms (Littke, 1993; Pasley et al., 1991, 1993).

According to Pasley et al. $(1991,1993)$, the concentrations of TOC and HI remain in general low in the regressive shelf events despite the relative increase in the deposition of terrestrial OM. However, in transgressive shelf events, the newly deposited rocks receive relatively little terrigenous sediments and $\mathrm{OM}$ since most of these materials remain trapped in the estuarine and coastal environments.

The reduction of the OM concentrations during shelf regressive events is associated with the largest degradation of organic fragments, resulting from the exposure of the sedimentary package to air during the fall in sea level.

Conversely, with a regrading shoreline during transgressive events, the areas of vegetation are slowing encroached by seawater. Aquatic organisms (phytoplankton and zooplankton) start to dominate the environment becoming the principal organic component. The accumulation and preservation of these organic aquatic 
constituents depends on numerous environmental parameters such as water circulation, productivity and depositional rates.

According to Emery and Myes (1996) during the LST, sediment influx bypassed the shelf and upper slope environments. The OM consisted of reworked terrestrial material deposited in distal fans, mainly in the form of oxidized inertinite or vitrinite. Many authors consider the TST as the most important stratigraphic sequence for the occurrence of source rocks or rich organic deposits (Emery and Myes, 1996). However, not all TST have rich-organic material. For the HST, Emery and Myes (1996) concluded that deposits of DOM are found in the basin depocenter only if dysoxic conditions occur. In the Sequence II of the study area, (Fig. 7), larger amounts of the inertinite group were observed in LST rather than TST. This pattern was observed in borehole 5-CA-81-RS and to a lesser extent in borehole 2TG-96-RS.

In the three studied boreholes, TOC contents were the highest in TST. In Sequences II and IV, the HST, shows a reduction in both TOC and vitrinite group with an increase of the inertinite group amounts as compared to the TST (boreholes 7-GT-10-RS and 2-TG-96-RS). This behavior could be associated with a relatively oxic depositional environment with a distal position in the basin.

Robinson and Engel (1993) studying a transgressive sequence, in Egypt, observed that the TST contained higher values of TOC and HI than the LST and HST. Similar results were observed by Pasley et al. (1993), Herbin et al. (1993), Triguis et al. (1996), Caplan and Bustin (1998) and WallaceDudley et al. (1998).

The HI in Sequence II showed higher concentrations in the TST than in LST and HST (boreholes 5-CA-81-RS and 2-TG-96-RS; Fig. 7).

The summarized distribution of the several measured parameters and their relation to the sequence stratigraphy of the study area is shown in Fig. 8. Terrestrial OM was the dominant material confirmed by microscopic analysis in all stratigraphic sequences in the study area. From pyrolysis this material was identified as Kerogen Types III and IV. The parameters of vitrinite, TOC and HI reflect the preservation conditions for each system tract. The relatively high concentration values of these parameters in the TST system tract indicate a less oxidant environment or a dysoxic zone. The reduction of inertinite and the increase in vitrinite supports this interpretation for the TST system tract. The LST and HST had oxic depositional conditions, evidenced by the reduction in concentrations of TOC, $\mathrm{HI}$ and vitrinite, and the corresponding increase in inertinite concentrations.

There is a small number of published analogues where the stratigraphic sequence contains a predominance of continental derived OM; however, assumptions in regards to oxidation of the organic materials based on geologic history, paleogeography and paleoclimate conditions should be valid regardless of the type of organic matter.

The basin position is the dominant factor in the LST, affecting the degree of oxygenation of the dominant terrestrial organic fragments of the inertinite or vitrinite group in the study area. Long transport of OM to the depositional site increased the action of weathering. The LST deposits contained reworked organic material that has been exposed to the atmosphere and were deposited in highenergy shallow water (Emery and Myes, 1996). The sea level drop reduced the effective depositional area concentrating these oxidized and reworked organic fragments in the LST.

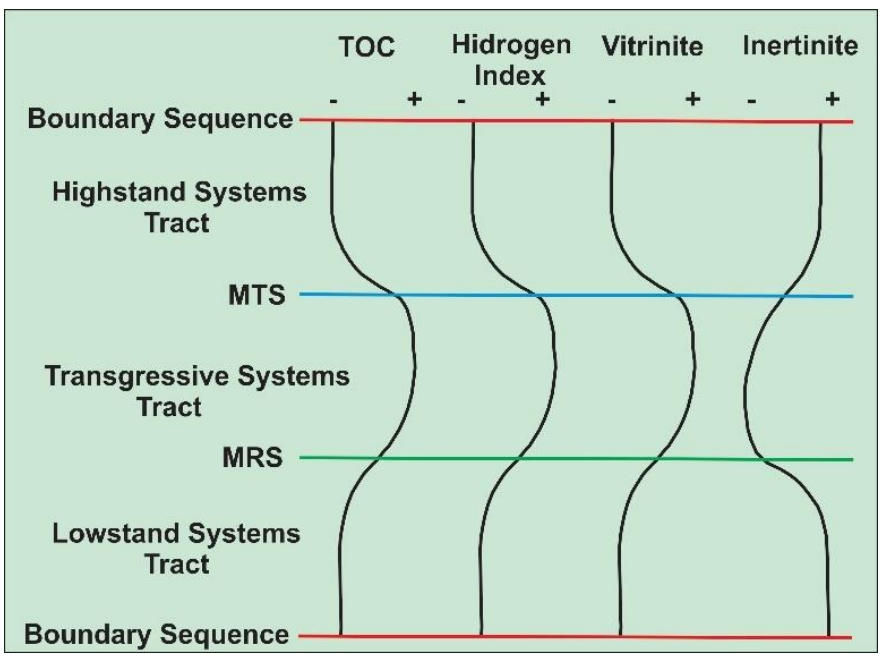

Fig. 8. Schematic diagram showing trend of general behaviour of organic parameters in sequence stratigraphic in the study area.

Conversely, during transgressive events or sea level rising, the depositional area is enlarged resulting in a more regional distribution of organic matter. Poor water circulation occurred in the deep basinal areas during the rise in sea level over the irregular geomorphology created by erosion during the previous regressive event. This circulation pattern created relatively dysoxic conditions that increased the accumulation and preservation of OM (Hay, 1995). This dysoxic depositional environment created the conditions for the transformation of woody material into vitrinite, increasing the vitrinite concentrations in TST. On the other hand, the transgressive event shifted the shoreline toward the continent and increased siliciclastic sedimentation. This increase in the sedimentation rate tended to reduce the deposited OM despite the better conditions for its preservation (Creaney and Passey, 1993).

In the HST, the reduction of the organic material concentration (TOC) is related to the increase of the water depth. The longer transport time to the deeper basinal areas in oxic conditions resulted in the degradation of the OM from exposure until it was incorporated into the sediment. In this case, the diagenetic resistant terrestrial organic fragments 
possibly had a better chance of survival and preservation. Consequently, the type of depositional system (forestepping or backstepping; Pasley et al., 1991 and 1993), the volume of sedimentary material and type of organic particle, influences the OM concentration. The influx of inorganic sediments during shoreline progradation causes dilution of OM concentrations. During transgression, with less sedimentation within the basin, larger percentages of $\mathrm{OM}$ are preserved. Associated with modifications of the depositional environment, the ratio of inertinite to vitrinite changes.

\section{Conclusion}

The petrography in reflected light of DOM, in the study area, indicates that the sedimentary rocks (shale, claystone and siltstone) contain predominately organic constituent materials of the inertinite and vitrinite group (continental $\mathrm{OM})$. The low values of the hydrogen and oxygen indexes analyzed through pyrolysis Rock-Eval III, indicate that the kerogen, in the study area, is of Type III and IV, not favorable for the generation of oil.

Nos estratos superiors à Rio Bonito Formation a OM encontra-se imatura mas atingiu a oil window (catagenesis) nos estratos localizados abaixo desta formação.

The OM maturation only reached the oil window (catagenesis) in strata inferior to the Rio Bonito Formation. In the sedimentary section above the Rio Bonito Formation was found immature OM, although in some places adjacent to intrusive rocks, metagenesis was reached.

The distribution of DOM in the analyzed boreholes evidenced relationships to several zones within the sequence stratigraphic framework. In general, TOC, HI and vitrinite concentrations are lower in the LST and HST than the TST. Conversely, the inertinite group has higher concentrations in the LST and HST than the TST. The liptinite group did not show a distribution pattern in the studied formations that could be used as a zonation parameter.

Dysoxic or oxic conditions at the time of deposition controlled the distribution and magnitude of OM within the studied formations. However, the distribution patterns for each system tract as related to the paleoenvironment and paleogeography will require further study.

The occurrence of Acritarchs in Sequence II in the LST and TST (Rio Bonito Formation) indicates the first marine transgression into the continental deposits of this Formation.

\section{Acknowledgment}

The Brazilian agencies Coordenação de Aperfeiçoamento de Pessoal de Nível Superior (CAPES) and Conselho Nacional de Desenvolvimento Científico e Tecnológico (CNPq) provided the financial support for this study. The authors thank the Compania de Pesquisa e Recursos Minerais
(CPRM) for supplying the samples from the boreholes and the well logs. Petróleo Brasileiro S.A. (PETROBRAS) provided the pyrolysis analysis and literature that was essential for this research. The authors would like to thank very much Professor Dr. Manuel João Lemos de Sousa of Universidade do Porto (Portugal), Professora Dra. Zuleika Carretta Corrêa da Silva of Universidade Federal do Rio Grande do Sul (UFRGS) and Dra. Maristela Bagatin Silva of da Universidade Federal de Pelotas (UFPel), for comments and suggestions which contributed to the improvement of study. The authors would like to thank Glenn Carpenter (Halliburton Energy Co.) for the reviews and constructive criticism.

\section{References}

Amaral, S.E., 1971. Geologia e petrologia da Formação Iratí (Permiano) no Estado de São Paulo. Boletim Instituto de Geociências e Astrononomia, São Paulo, 2, 3-81.

Araújo, L.M., Triguis J.A., 1994. Evaluation of hydrocarbon generation processes of the Iratí Formation source rock Paraná Basin, Brazil, $4^{\text {th }}$ Latin American Congress on Organic Geochemistry- ALAGO, Bucaramanga-Colombia. Abs. p. 92.

Bostick, N.H., 1979. Microscopic measurement of the level of catagenesis of solid organic matter in sedimentary rocks to aid exploration for petroleum and to determine former burial temperatures - a review. Society of Economic Paleontologists and Mineralogists, Special publication, 26, 17-43.

Brooks, J., 1981. Organic Maturation Studies and Fossil Fuel Exploration. Academic Press Ind., London.

Burjack, M.I.A., 1984. Caracterização da matéria orgânica dispersa nos sedimentos da Formação Iratí. Permiano superior da Bacia do Paraná, Porto Alegre. PhD thesis, Instituto de Geociências, Universidade Federal do Rio Grande do Sul, 231p. Unpublished.

Calvert, S.E., Pedersen, T.F., 1993. Geochemistry of Recent oxic and anoxic marine sediments: implications for the geological record. Marine Geology, 113, 67-88. doi.org/10.1016/00253227(93)90150-T

Caplan, M.L., Bustin, R.M., 1998. Sedimentology and sequence stratigraphic of Devonian-Carboniferous strata, south Alberta. Bulletin of Canadian Petroleum Geology, 46 (4), 487-514.

Castaño, J.R., Sparks, D.M., 1974. Interpretation of vitrinite reflectance measurements in sedimentary rocks and determination of burial history using vitrinite reflectance and authigenic minerais. Special Paper of the Geological Society of America 153, 31-52. doi: 10.1130/SPE153-p31

Cerqueira, J.R., Santos Neto, E.V., 1986. Projeto análise da Bacia do Paraná (Geoquímica Orgânica). Relatório interno CENPES/SINTEP, nº 673 - 9014, 3 vol., Rio de Janeiro.

ICCP, 1993. Visual classification used by commission II. In: Intemational Committee for Coal Petrology, unpublished.

Corrêa da Silva, Z.C., Cornford, C., 1985. The kerogen type, depositional environment and maturity of the Irati Shale, Upper Permian of Paraná Basin, Southern Brazil. Organic 
Geochemistry, 8(6), 399-411. doi.org/10.1016/01466380(85)90018-X

Creaney, S., Passey, Q.R., 1993. Recurring patterns of total organic carbon and source rock quality within a sequence stratigraphic. American Association of Petroleum Geologists, AAPG Bulletin, 77(3), 386-401.

DNPM/CPRM, 1986. Projeto A borda leste da Bacia do Paraná. Integração geológica e avaliação econômica. Porto Alegre, 18v (Relatório interno).

Emery, D., Myers, KJ. 1996. Organic-rich facies and hydrocarbon source rocks. In: Emery, D. and Myers, KJ., (eds.). Sequence stratigraphy. Blackwell Science, pp. 238-257.

Espitalié, J., Deroo, G., Marquis, F. 1985. La pyrolyse Rock-Eval et ses applications. Oil \& Gas Science and Technology - Rev. IFP, 40(6), 755-784. doi.org/10.2516/ogst:1985035

Etgeton, W., 1997. Aplicação dos conceitos de estratigrafia de seqüências ao intervalo permiano eotriássico da Bacia do Paraná na região nordeste do Rio Grande do Sul - Brasil. M.Sc dissertation, Instituto de Geociências, UFRGS, unpublished.

Goulart, E.P., Jardim, N.S., 1982. Avaliação geoquímica das formações Ponta Grossa e Iratí - Bacia do Paraná. In: Consórcio CESP/IPT. Geologia da Bacia do Paraná. Reavaliação da potencialidade e prospectividade em hidrocarbonetos. São Paulo.

Hay, W.W., 1995. Paleoceanography of Marine Organic-CarbonRich Sediments. In: Paleogeography, Paleoclimate, and Source Rocks., ed. By Huc, Alain-Yves. Studies in Geology, 40. American Association of Petroleum Geologists, Tulsa, pp. 21 59. ISBN 0-89181-048-X

Herbin, J.P., Müller, C., Jeissant, J.R., Méliéres, F., Penn, I.E., Group, Y., 1993. Variation of the distribution of organic matter within a transgressive system tract: Kimmeridge clay (Jurassic), England. In: Katz, B.J. and Pratt, L. M.(eds.). Source rocks in a sequence stratigraphic framework. Am. Assoc. Petr. Geol., Study in Geology 37, 67-100.

Hoffman. E., Jenkner, A. (1932). Die Inkohlung und ihre Erkennung im Mikrobildung. Gluckauf, 68, 81 p.

Holz, M. 1997. Early Permian sequence stratigraphy of the Paraná Basin in northeastern Rio Grande do Sul state, Brazil. Anais da Academia Brasileira de Ciências, 69(4), 521-543.

Holz, M., Picolli, A.E., Vieira, P.E., 1986. Análise estratigráfica das bacias carboníferas no nordeste do estado do Rio Grande do Sul: Gravataí Morungava, Chico Lomã e Santa Terezinha. In: Congresso Brasileiro de Geologia, 34, Goiânia, 1986. Anais,1, 177-190.

Hutton, A., Burgess, J., Stasiuk, L., Cardou, B. and ThompsonRizer, C., 1999. TSOP Research Committee Progress Report: ICCP and TSOP Join Forces on Kerogen Classification. TSOP Newsletter, 16(1), 21-25.

Jones, R.W., 1987. Organic faces. In: Brooks J., Welte O. (eds.). Advance in Petroleum Geochemistry, London, Academic Press, 2, 1-90.

Larsen, G., Chilingar, G.V., 1983. Diagenesis in Sediments and Sedimentary Rocks. Volume 2. Diagenesis, Elsevier, 572 p.
Katz, B.J., 1983. Limitations of Rock-Eval pyrolysis for typing organic matter. Organic Geochemistry, 4(3), 195-199. doi.org/10.1016/0146-6380(83)90041-4

Lavina, E.L., Lopes, R.C. 1987. A transgressão marinha no Permiano inferior e a evolução paleogeográfica do Supergrupo Tubarão no Estado do Rio Grande do Sul. Paula-Coutiana, Porto Alegre, 1, 51-103.

Littke, R., Baker D.R., Rullkötter J., 1997. Deposition of Petroleum Source Rocks. In: Welte, D.H., Horsfield, B., Baker, D.R. (eds), Petroleum and Basin Evolution, Springer, Berlin, Heidelberg. doi.org/10.1007/978-3-642-60423-2_6

Littke, R., 1993. Deposition of organic matter-rich sediments. In: Littke, $\mathrm{R}$ (ed.). Deposition, diagenesis and weathering of organic matter-rich sediments. Lecture Notes in Earth Science, $47,12-45$.

Lo, H.B., 1992. Identification of indigenous vitrinites for improved thermal maturity evaluation. Organic Geochemistry, 18(3), 359364.

McCartney, J.T., 1952. A study of the Seyler theory of coal reflectance: Economic Geology 47 (2), 202-210.

Milani, E.J., Melo, J.H.G., Souza, P.A., Fernandes, L.A., França, A.B., 2007. Bacia do Paraná. Boletim de Geociências da Petrobras, Rio de Janeiro, 15(2), 265-287.

Mukhopadhyay, P.K., 1994. Vitrinite reflectance as maturity para meter. In: Mukhopadhyay, P.K. and Dow, W.G. (eds.). Vitrinite Reflectance as Maturity Parameter, American Chemical Society, Washington, pp.1-24. ISBN: 9780841229945

Parrish, J.T., 1995. Paleogeography of Corg-rich rocks and the preservation versus production controversy. In: Huc, A.Y.(ed.), Paleogeography, paleoclimate, and source rocks. The American Association of Petroleum, Study in Geology, 40, 1-20.

Pasley, M.A., Gregory, W.A., Hart, G., 1991. Organic matter variations in transgressive and regressive shales. Organic Geochemistry, 17(4), 483-509.

Pasley, M.A., Ríley, G.W., Nummedal, D., 1993. Sequence stratigraphic significance of organic matter variation: Example from upper Cretaceous Marcos Shale of the San Juan Basin, New Mexico. In: Katz, B.J. and Pratt, I.M.(eds.), Souree rocks in a sequence stratigraphic framework, The American Association of Petroleum, 37, 221-241.

Peters, K.E., 1986. Guidelines for evaluating petroleum source rock using programmed pyrolysis. The American Association of Petroleum, AAPG Bulletin, 70(3), 318-329.

Piccoli, A.E.M., Lopes, R.C., Camozzato, E., 1986. Análise paleoambiental das jazidas de carvão do Estado do Rio Grande do Sul. Acta Geológica Leopoldensia, São Leopoldo, 22 (10), 35-54.

Robert, P., 1988. Organic Metamorphism and geothermal history: Microscopic study of organic matter and thermal evolution of sedimentary basins. Reidel, Dordrecht, 311pp.

Robinson, V.D., Engel, M.H., 1993. Characterization of the source horizons within the late Cretaceous transgressive sequence of Egypt. In: Katz, B.J and Pratt, L. M.(eds.), Source rocks in a sequence stratigraphic framework. American Association of Petroleum Geologists, Study in Geology, 37, 101-118. 
Schneider, R.L., Muhlmann, H., Tommasi, E., Medeiros, R.A., Daemon, R.F., Nogueira, A.A., 1974. Revisão estratigráfica da bacia do Paraná. 28 ${ }^{\text {th }}$ Congresso Brasiliero de Geologia, Porto Alegre, 1, 41-65.

Senftle, J.T., Landis, C.R., 1991. Vitrinite reflectance as a tool to assess thermal maturity. In: Merrill R.K.(ed.), Source and Migration Processes and Evaluatíon Techniques, Treatise of Petroleum Geology, Handbook of Petroleum Geology, American Association of Petroleum Geologists, Tulsa, p.119125.

Stach, E., Mackowsky, M.T., Teichmüller, M., Teichmuller, R., Taylor, G.H., Chandra, D., 1975. Stach's Textbook of Coal Petrology. $3^{\text {rd }}$ ed. Gebrüder Borntrager, Berlim-Stuttgart.

Taylor, G.H., Teichmüller, M., Davis, A., Diessel, C.F.K., Littke, R., Robert, P., 1998. Organic Petrology. Gebrüder Borntraeger, Berlin, Stuttgart.

Teerman, S.C., Cardou, B.J., Harding, R.W., Lemos de Sousa, M.J., Logan, D.R., Pinheiro, H.J., Reinhard, M., Thompson-Rizer, C.L., Woods, R.A., 1995. Source rocks/dispersed organic matter characterization - TSOP. Research Subcommittee Results. Organic Geochemistry, 22 (1), 11-25.

Teichmüller, M., 1987. Recent advances in coalification studies and their application to geology. In: Scott, A.(ed.). Geological Society of London, Public Special, London, 32, 127-169.

Teichmüller, M., 1986. Organic petrology of source rocks, history and state of the art. In: Leythaeuser, D. and Rullkõtter, J. (eds.), Advances in Organic Geochemistry 1985, Part I, Petroleum Geochemistry (Proc. Internat. Meet Organ. Geochem., 12th Jülich, 1985). Organic Geochemistry, 10, (1/3): pp.581-599.

Teichmüller, M., 1975. Rank determination on sedimentary rocks other than coal. In: Stach E., Mackowsky, M.T., Teichmüller, M., Teichmüller, R., Taylor, G.H., Chandra D. (eds.). Textbook of Coal Petrology, $2^{\text {nd }}$ ed., p. 303-309.
Tissot, B.P., Welte, O.H., 1978. Petroleum Formafion and Occurrence. Springer Verlag, Berlin, Heildelberg.

Triguis J., Araújo, L.M., França, B., Winter, W.R., 1996. Deposicional environment and sequence stratigraphy of the Irati Formation (Iate Permian) Paraná Basin- Brazil. 5th Latin American Congress on Organic Geochemistry - ALAGO, Cancun, México.

Tyson, R.V., 1987. The genesis and palinofacies characteristics of marine petroleum source rocks. In: Brooks, J. and Fleet, A.J.(eds.). Marine Petroleum Source Rocks. Geological Society of London Special Publication, 26, 47-67.

Tyson, R.V., Pearson, T.H., 1991. Modern and ancient continental shelf anoxia: an overview. In: Tyson, R.V. and Pearson, T.H. (eds.), The Geological Society Special Publication, Oxford, 58:1-24.

van Krevelen, D.W., 1961. Coal: Typology - Chemistry - PhysicsConstitution, 1st ed. Elsevier, Amsterdam. 514 pp.

Wallace-Oudley, K.E., Leckie, O.A., Vanbeselaere, N.A., James, P.O., 1998. Regional geology, sedimentology and sequence stratigraphic framework of the Albian Pense Formation southwestern Alberta. Bulletin of Canadian Petroleum Geology, 46(4), 599-632.

Wang, L., 1989. Detailed investigation of the pyrolysis process and new applications of the technique in organic geochemistry. PhD Thesis, Univ. of Tulsa.

White, I.C. 1908. Relatório sobre as camadas de carvão e rochas associadas do sul do Brasil. Rio de Janeiro. Final Report Comission, Est. Minas de Carvão de Pedra do Brasil, 300 pp.

Zalán, PV., Wolff, S., Conceição, J.C., Marques, A, Astolfi, M.AM, Vieira, I.S., Appi, V.T., Zanotto, O.A., 1990. Bacia do Paraná. In: Gabaglia, R.G.P., Milani, E.J.(eds.), Origem e evolução das bacias sedimentares, Petrobrás, p. 135-168. 